\title{
On the dynamics of the $\mathrm{H}^{+}+\mathrm{D}_{2}(v=0, j=0) \rightarrow \mathrm{HD}+\mathrm{D}^{+}$reaction: A comparison between theory and experiment
}

\author{
Estela Carmona-Novillo, Tomás González-Lezana, ${ }^{\text {a) }}$ and Octavio Roncero \\ Instituto de Matemáticas y Física Fundamental (CSIC), Serrano 123, 28006 Madrid, Spain \\ Pascal Honvault \\ Institut UTINAM, UMR CNRS 6213, University of Franche-Comté, Campus de la Bouloie, \\ 25030 Besançon Cedex, France \\ Jean-Michel Launay \\ PALMS, UMR CNRS 6627, University of Rennes 1, Campus de Beaulieu, 35042 Rennes Cedex, France \\ Niyazi Bulut, ${ }^{\text {b) }}$ F. Javier Aoiz, and Luis Bañares \\ Departmento de Química Física I, Facultad de Química, Universidad Complutense, 28040 Madrid, Spain
}

Alexandre Trottier and Eckart Wrede

Department of Chemistry, University of Durham, Durham DH1 3LE, United Kingdom

(Received 21 September 2007; accepted 22 October 2007; published online 3 January 2008)

The $\mathrm{H}^{+}+\mathrm{D}_{2}(v=0, j=0) \rightarrow \mathrm{HD}+\mathrm{D}^{+}$reaction has been theoretically investigated by means of a time independent exact quantum mechanical approach, a quantum wave packet calculation within an adiabatic centrifugal sudden approximation, a statistical quantum model, and a quasiclassical trajectory calculation. Besides reaction probabilities as a function of collision energy at different values of the total angular momentum, $J$, special emphasis has been made at two specific collision energies, 0.1 and $0.524 \mathrm{eV}$. The occurrence of distinctive dynamical behavior at these two energies is analyzed in some detail. An extensive comparison with previous experimental measurements on the Rydberg $\mathrm{H}$ atom with $\mathrm{D}_{2}$ molecules has been carried out at the higher collision energy. In particular, the present theoretical results have been employed to perform simulations of the experimental kinetic energy spectra. (C) 2008 American Institute of Physics.

[DOI: $10.1063 / 1.2812555]$

\section{INTRODUCTION}

In recent experimental works, ${ }^{1,2}$ the dynamics of the $\mathrm{H}^{*}(n)+\mathrm{D}_{2} \rightarrow \mathrm{HD}+\mathrm{D}^{*}(n)$ reaction was investigated in detail. It was found that this process, in which the hydrogen atom is in a highly excited Rydberg state with $n \sim 45-50$, presents strong similarities to the ion-diatom $\mathrm{H}^{+}+\mathrm{D}_{2} \rightarrow \mathrm{HD}+\mathrm{D}^{+}$reaction. In particular, the rotationally resolved product distributions for the Rydberg-atom reaction was in a good agreement with that obtained at a single scattering angle for the ionatom reactive collision. The equivalence between both processes could be explained considering that the electron of the Rydberg atom effectively behaves as a spectator, in such a way that, during the collision, the hydrogen atom participates in essence as an ion. One of the main implications of these findings is that Rydberg $\mathrm{H}$-atom translational spectroscopy can be a practical alternative to investigate ion-molecule reactive processes.

Quasiclassical trajectory (QCT) calculations on the $\mathrm{H}^{+}$ $+\mathrm{D}_{2} \rightarrow \mathrm{HD}+\mathrm{D}^{+}$reaction revealed, however, that the experimental differential cross section (DCS) obtained for the Rydberg-atom reaction exhibited some asymmetry in the

\footnotetext{
a) Author to whom correspondence should be addressed. Electronic mail: tglezana@imaff.cfmac.csic.es

${ }^{b}$ Present address: Firat University, Department of Physics, 23169 Elazig, Turkey.
}

forward-backward scattering directions, which was not seen in the theoretical distribution. ${ }^{3}$ Quite recently, Hayes and Skodje $^{4}$ have attributed these qualitative differences between both processes to effects due to some dynamics of the Rydberg electron. Since only product Rydberg atoms with $n$ $\geqslant 20$ are detected in the experiment, and the $\mathrm{H}$ atom is pumped to a highly excited Rydberg state $(n=50)$ before the collision takes place, losses of experimental signal can be interpreted as an indication of either relaxation or ionization phenomena of the Rydberg atom. The authors of Ref. 4 concluded that at least part of the asymmetry found in the measured DCSs could be due to some angle dependent attenuation of the signal detected on the experiments.

The potential energy surface (PES) corresponding to $\mathrm{H}_{3}^{+}$ exhibits a crossing between the energy curves of the electronic states correlating to $\mathrm{H}^{+}+\mathrm{H}_{2}$ and $\mathrm{H}_{2}^{+}+\mathrm{H}$ at about $1.6 \mathrm{eV}$ above the $\mathrm{H}_{2}$ well. A large deal of work has addressed the dynamics of the ion-diatom reactions for the different isotopic variants of this system at energies far above this crossing, studying thus the competition among the possible reaction pathways: (i) the reactive noncharge transfer (RNCT) channel or atomic-ion formation, in which the charge remains at the atom; (ii) the nonreactive charge transfer channel, in which the process leads to the migration of the charge from the ion to the diatom; and (iii) the reactive 
charge transfer channel, in which the charge ends in the product diatom after a charge transfer plus rearrangement process.

Most of these previous studies have been carried out by means of trajectory calculations ${ }^{5-15}$ or quantum mechanical (QM) calculations at zero total angular momentum $(J=0) .{ }^{13,15,16}$ Marković and Billing ${ }^{17}$ employed a semiclassical wave packet method to calculate cross sections for the nonadiabatic $\mathrm{D}^{+}+\mathrm{H}_{2}$ reaction under a coplanar approximation which neglects the Coriolis coupling terms. One of the main reasons for these limitations is the existence of a deep potential well (about $4.3 \mathrm{eV}$ ), which complicates the application of exact quantum mechanical (EQM) methods to study the dynamics of these systems. In fact, only recently results from EQM calculations have been reported for the $\mathrm{D}^{+}+\mathrm{H}_{2}$ (Ref. 18) and $\mathrm{H}^{+}+\mathrm{D}_{2}$ (Ref. 19) reactions. For the low energy regime $\left(E_{c}<1.6 \mathrm{eV}\right)$, below the electronic curve crossing, at which the RNCT channel constitutes the only reaction pathway and nonadiabatic effects can be discarded, a quantum wave packet (QWP) approach and a time independent (TI) EQM method were employed to tackle the study of the $\mathrm{H}^{+}+\mathrm{D}_{2},{ }^{20} \mathrm{D}^{+}+\mathrm{H}_{2},{ }^{20}$ and $\mathrm{H}^{+}+\mathrm{H}_{2}$ (Ref. 21) reactions.

Much has been reported about the dynamics of the different isotopic variants of the ion-diatom collisions associated to the $\mathrm{H}_{3}^{+}$system. It has been proposed that this sort of collisions may be mediated by the formation of an intermediate complex, specially in the low energy regime. This mechanism is certainly consistent, in principle, with the above mentioned potential well and the absence of any barrier at the entrance channel. However, initial suggestions in this sense regarding the $\mathrm{D}^{+}+\mathrm{H}_{2}$ process ${ }^{22}$ seemed not to be unquestionably corroborated in subsequent studies for some other isotopic analogs. ${ }^{23,24}$ Holliday et al. $^{25}$ concluded from the energy distributions of product and reactant ions that despite the collisions could be interpreted as result of a "complex formation" at relatively low collision energies and "direct reaction" at higher energies for the RNCT processes, a complete description in terms of a competition between these two reaction pathways is shown to be an oversimplification. This apparent transition from one dynamical mechanism to the other was also suggested in different trajectory calculations. ${ }^{6-9,11,26}$ Thus, the complex-formation cross sections for the $\mathrm{D}^{+}+\mathrm{D}_{2},{ }^{11,26} \mathrm{D}^{+}+\mathrm{H}_{2},{ }^{11,26} \mathrm{H}^{+}+\mathrm{H}_{2},{ }^{9,11}$ and $\mathrm{H}^{+}$ $+\mathrm{D}_{2}$ reactions ${ }^{7,11,26}$ were found to decrease rapidly as the collision energy increases. ${ }^{11}$ In addition, lifetimes of the intermediate complexes also shortened when the energy was augmented. ${ }^{7}$

This dynamical picture for the ion-diatom $\mathrm{H}_{3}^{+}$collisions would explain the use of statistical techniques in previous occasions. Takayanagi et al. ${ }^{13}$ found that cumulative reaction probabilities for $J=0$ for the $\mathrm{RNCT} \mathrm{D}^{+}+\mathrm{H}_{2} \rightarrow \mathrm{DH}+\mathrm{H}^{+}$reaction could be reproduced fairly well by means of a statistical estimation based on the effective number of available states below $2.1 \mathrm{eV}$ total energy. The authors interpreted the large number of resonances existing in the reaction probability as an indication of absence of direct reaction in that energy range. A similar analysis on the $\mathrm{DH}_{2}^{+}$system for $J=0$ (Ref. 16) revealed that the statistical prediction also gives a good estimate of the total cumulative reaction probabilities for the $\mathrm{H}^{+}+\mathrm{HD} \rightarrow \mathrm{H}_{2}+\mathrm{D}^{+}$process, the only purely adiabatic reaction of the system, whereas the other possible collisions involving two $\mathrm{H}$ atoms and one $\mathrm{D}$ atom, which imply nonadiabatic transitions, exhibited significant deviations from a statistical behavior. In particular, since the rearrangements of atoms can proceed only through the lower PES (because of the high barrier existing at the upper PES), reaction probabilities for the other RNCT collision studied in Ref. 16, namely, $\mathrm{H}+\mathrm{HD}^{+} \rightarrow \mathrm{H}_{2}^{+}+\mathrm{D}$, with a nonadiabatic transition in both reactants and product channels, were clearly not reproduced by the statistical approach.

The rotational distributions for the $\operatorname{HD}\left(v^{\prime}=0, j^{\prime}\right)$ fragments obtained in the experiments on the Rydberg-atom $\mathrm{H}^{*}+\mathrm{D}_{2}$ reaction by Wrede et al. ${ }^{2}$ at the collision energy of $E_{c}=0.53 \mathrm{eV}$ displayed a roughly statistical profile. In particular, the relative rotational HD populations extracted from the kinetic energy spectra (KES) measured at two different laboratory scattering angles $\left(\theta_{\mathrm{lab}}=7.5^{\circ}\right.$ and $\left.15^{\circ}\right)$ were compared with prior distributions and the agreement achieved was in general good. Although pronounced deviations from the statistical predictions were evident for some of the higher rotational levels (as $j^{\prime}=8$ ), the HD product states were mainly statistically populated. DCSs calculated by means of a statistical quantum method, ${ }^{27,28}$ (SQM) for the corresponding ion-diatom reaction, $\mathrm{H}^{+}+\mathrm{D}_{2}$, at $E_{c}=0.524 \mathrm{eV}$, were also found in good agreement ${ }^{20}$ with the measured distributions of Ref. 1, in the angular range covered by the experiment.

More recently, on a study on the $\mathrm{H}^{+}+\mathrm{H}_{2}$ reaction at $E_{c}$ $=0.44 \mathrm{eV}$ performed by some of $\mathrm{us}^{21}$ the same SQM approach yielded results in good agreement with reaction probabilities, integral cross sections (ICSs), and DCSs obtained with an EQM calculation. The only relevant discrepancies with the exact results were found at the state-to-state level for some DCSs and for opacity functions corresponding to transitions to high product rotational states, $\mathrm{H}_{2}\left(v^{\prime}=0, j^{\prime}\right.$ $\geqslant 4$ ), for which the SQM predictions end at much lower values of $J$ than both the exact and QCT probabilities. It has been shown recently, ${ }^{29}$ however, that the origin of the discrepancies is the use of the centrifugal sudden (CS) approximation in the application of the SQM. The differences with the exact results disappear almost completely when the rigorous coupled-channel (CC) version of the statistical model is used. Also in accord with this possible transition from a complex-forming dynamics to a predominantly direct impulsive reaction pathway as the collision energy increases is the failure of a phase-space theory approach to reproduce molecular beam experiment cross sections at relative energies above $2 \mathrm{eV}{ }^{6}$ The statistical results were found to yield cross sections in clear disagreement in both absolute and relative senses in comparison with QCT and experimental results. Moreover, the product branching ratios were not correct.

One of the most intriguing results from our previous study on the $\mathrm{H}^{+}+\mathrm{H}_{2}$ reaction is the apparent limitations of the QCT approach to provide a good description of the dynamics for high values of the total angular momentum. The opacity function or reaction probability in terms of $J$ at $E_{c}$ $=0.44 \mathrm{eV}$ shows that the QCT values remain clearly below the EQM result as total angular momentum increases. In ad- 
dition, the trajectory approach fails to reproduce the extremely peaked profile of the exact DCSs at the forward $(\theta$ $\left.\sim 0^{\circ}\right)$ and backward $\left(\theta \sim 180^{\circ}\right)$ scattering directions. Interestingly, the application of a recently developed QCT version of the SQM model of Refs. 27 and 28 has revealed that differences between results produced with both statistical approaches for the $\mathrm{H}^{+}+\mathrm{H}_{2}$ and $\mathrm{H}^{+}+\mathrm{D}_{2}$ reactions are, on the contrary, negligible. ${ }^{29,30}$ Considering the good description of the EQM results achieved by the SQM model for the $\mathrm{H}^{+}$ $+\mathrm{H}_{2}$ process, $^{21}$ it is then possible to conclude that QCTbased methods can also be capable to explain the dynamical mechanisms of these ion-diatom collisions. The origin of the previously mentioned deficiencies of the QCT approach should not be therefore necessarily attributed to the existence of purely QM effects in the process.

Although the explicit inclusion of parity conservation $^{29-32}$ certainly solves the issue of the forward/ backward peaks of the DCSs, the opacity functions obtained by means of the version of the statistical quasiclassical trajectory (SQCT) model when ignoring such parity conservation effects for both the $\mathrm{H}^{+}+\mathrm{H}_{2}$ and $\mathrm{H}^{+}+\mathrm{D}_{2}$ reactions do not deviate excessively from the SQM prediction, in clear disagreement with the usual QCT approach. In that sense, it is interesting to indicate the differences of up to $40 \%$ found between trajectory calculations and the Rice-RamspergerKassel-Marcus (RRKM) predictions for the unimolecular decomposition of the $\mathrm{HD}_{2}^{+}$system. ${ }^{33}$ Authors of Ref. 33 observed that the magnitude and the sign of the discrepancy strongly depend on the value of $J$. RRKM and trajectory estimates for the mean lifetime of the complex were brought into accord by means of a correction factor to the statistical formula to account for direct trajectories.

In the present work, we have carried out a comprehensive study of the $\mathrm{H}^{+}+\mathrm{D}_{2}(v=0, j=0) \rightarrow \mathrm{HD}+\mathrm{D}^{+}$reaction dynamics by means of TI EQM and QWP approaches on the $a b$ initio PES of Aguado et al. ${ }^{34}$ As in our previous paper on the isotopic variant $\mathrm{H}^{+}+\mathrm{H}_{2}(v=0, j=0)$, results obtained with these QM calculations are compared with SQM and QCT predictions. Comparisons with the experiments of Dai et al. ${ }^{1}$ and Wrede et $a l^{2}{ }^{2}$ on the Rydberg $\mathrm{H}$-atom reaction with $\mathrm{D}_{2}$ molecules at $E_{c} \approx 0.53 \mathrm{eV}$ collision energy have been made. In particular, simulations of the experimental KES at different laboratory angles by using the EQM, SQM, and QCT state-to-state DCSs have been carried out. Furthermore, the theoretical study has been extended to the lower collision energy $E_{c}=0.1 \mathrm{eV}$ to investigate possible changes in the dynamical mechanisms governing the reaction.

The paper is structured as follows: in Sec. II, details on the different computational methods are briefly described. In Sec. III, a concise description of the experiment is given. Finally, results are commented and discussed in Secs. IV and $\mathrm{V}$; the concluding remarks are shown in Sec. VI.

\section{THEORY}

\section{A. Time independent exact quantum method}

We have carried out EQM calculations using a TI method based on body-frame hyperspherical democratic coordinates. This method was presented in detail in Ref. 35 and has already been used for the isotopic variant $\mathrm{H}^{+}+\mathrm{H}_{2} .{ }^{21}$ This method has also previously proved successful in describing the quantum dynamics of atom-diatom insertion reactions, such as $\mathrm{N}\left({ }^{2} D\right)+\mathrm{H}_{2},{ }^{36}$ and ultracold alkali-dialkali collisions. ${ }^{37}$ Thus, a brief summary will suffice here. At each hyper-radius, the scattering wave function is expanded on a set of hyperspherical adiabatic states of a reference Hamiltonian $H=T+V$, which incorporates the kinetic energy $T$ arising from deformation at fixed hyper-radius and the potential energy $V$. The expansion coefficients are the solution of a set of coupled second-order differential equations, which are solved using the Johnson-Manolopoulos log-derivative propagator. $^{38}$ In this work, for the total angular momentum $J=0,211$ states dissociate at large hyper-radius into the $\mathrm{D}_{2}$ $(28,26,24,22,20,16,14,10,6)$ rovibrational set (this notation indicates the largest rotational level $j$ for each vibrational manifold $v=0,1, \ldots, 8)$ and the $\operatorname{HD}(23,21,19,16,14,11,8)$ rovibrational set. Propagation goes from $0.5 a_{0}$ up to the asymptotic matching distance of $16 a_{0}$, where the $S$ matrix is extracted. When computing $J>0$ partial waves, we have considered the $|\Omega|$ components from 0 to 28 in the closecoupling expansion to obtain accurate integral and differential cross sections. Thus, the number of coupled equations increases from 211 for $J=0$ to 2211 for $J>28$.

\section{B. Quantum wave packet method}

Exact QWP calculations in this system are extremely difficult because of the presence of many long lived resonances and the necessity of including many helicity components to obtain converged results. ${ }^{20,21}$ TI calculations are by far more efficient when only few collision energies are of interest. Nevertheless, approximate QWP calculations are performed here to bring some help to elucidate the complex dynamics of the title reaction. The adiabatic centrifugal sudden (ACS) approximation used in the present work consists in considering a single helicity projection, replacing the centrifugal barrier by the lower possible one for a given total angular momentum $J$ and a rotational state $j$ of the $\mathrm{D}_{2}$ reactant. ${ }^{20,21}$ Such approach allows to describe correctly the reaction threshold at moderate $J$ values ${ }^{20,21}$ at the same computational cost required for a $J=0$ calculation. In any treatment in which the helicity components for a particular Jacobi coordinate set are restricted to a finite value, the equivalence among symmetric rearrangement channels is broken. Using the ACS approach this symmetry is warranted.

The QWP approach within the ACS method (called simply QWP, hereafter) has been described previously, ${ }^{20}$ and only some relevant details are given here. The wave packet corresponding to a single effective helicity component is represented in numerical grids of the internal reactant Jacobi coordinates formed by $512 \times 640 \times 80$ for $r, R$, and $\gamma$, respectively. The equispaced radial points are considered in the intervals $0.3 \leqslant r \leqslant 25 \AA$ and $0.01 \leqslant R \leqslant 17.5 \AA$ while for $\gamma$, Gauss-Legendre quadrature points are used within the $[0, \pi / 2]$ interval, to account for the $\mathrm{D}_{2}$ permutation symmetry. Finally, to avoid artificial reflections at the end of the 
radial grids, the wave packet is multiplied by absorbing functions for $r \geqslant 21 \AA$ and $R \geqslant 13.5 \AA$, as described previously. ${ }^{20}$ A modified Chebyshev propagator is used to propagate a real wave packet. ${ }^{39-44}$ The initial real wave packet consists of the product of the rovibrational state of the reactant, $\mathrm{H}_{2}(v=0, j$ $=0$ ) in the present case, times the real part of an incoming Gaussian function. ${ }^{44}$

Total reaction probabilities are obtained by the method of flux for $J=0,5,10,15,20,25,30,35,40$, and 45 on a dense energy grid. At intermediate $J$ 's, the total reaction probabilities are obtained by weighted interpolation, using the calculated reaction probabilities, $P^{J}\left(E_{c}\right)$, and a modified $J$-shift approach.

State-to-state reaction probabilities are calculated in the QWP approach with a method previously described using reactant Jacobi coordinates. ${ }^{45}$ The choice of reactant Jacobi coordinates is made for two reasons. First, it allows an easy description of the $\mathrm{D}_{2}$ permutation symmetry. Second, a single helicity component $(\Omega=0$ for initial $j=0$ considered in this case) is well suited to describe the entrance channel for any $J$ value, whereas this would not be the case when using product Jacobi coordinates. ${ }^{45}$ The method used to extract the state-to-state probabilities consists in transforming the wave packet to product Jacobi coordinates at each iteration. The transformation is performed in several steps using option A of Ref. 45, thus reducing significantly the computational effort. Moreover, the flux for each $\operatorname{HD}\left(v^{\prime}, j^{\prime}\right)$ state is analyzed at only two $R^{\prime}$ values $(\approx 15 \AA)$ of product Jacobi coordinates. The transformation procedure takes then a negligible computational cost as compared to that of the propagation. However, for a proper description of the product states, the grids used are considerably larger than those required to calculate only total reaction probabilities.

High values of $J$ require special attention because the rotational barriers are not negligible at the product Jacobi distance $R^{\prime}=15 \AA$. Therefore, the typical exponential asymptotic behavior cannot be assumed, as it is done in the method of Balint-Kurti et al., ${ }^{46}$ as described in Ref. 45. Instead, the flux is analyzed here using two distances. Also, the flux towards all $\Omega^{\prime}$ channels should be included to get convergence and due to some peculiarities of the ACS approach. The convergence is checked by comparing with the total reaction probability obtained with the method of flux in reactant Jacobi coordinates. Because of the high density of resonances, the reaction probabilities vary a lot with energy and the error may be relatively large (below 10\%) at some narrow peaks, but in average it is always better than $1 \%$.

The state-to-state probabilities were calculated at the $J$ values mentioned above. An interpolation procedure, as that used for total reaction probabilities, is done for intermediate $J$ values. Because the dense manifold of resonances are present at all $J$ 's, the phase of the $S^{J}$ matrix elements are expected to vary fast, thus eliminating the interference effects. For this reason, the random phase approximation is used to obtain the state-to-state DCS as

$$
\begin{aligned}
\sigma_{v j \rightarrow v^{\prime} j^{\prime}}(E, \theta) \approx & \frac{1}{k_{v j}} \sum_{\Omega=-j}^{j} \sum_{\Omega^{\prime}=-j^{\prime}}^{j^{\prime}} \sum_{J=\min \left(\Omega, \Omega^{\prime}\right)}[(2 J+1) \\
& \left.\times d_{\Omega \Omega^{\prime}}^{J}(\theta)\right]^{2}\left|S_{v j, v^{\prime} j^{\prime}}^{J}(E)\right|^{2},
\end{aligned}
$$

where $d_{\Omega \Omega^{\prime}}^{J}(\theta)$ is a reduced rotation matrix ${ }^{47}$ and $k_{v j}$ $=\sqrt{2 \mu\left(E-E_{v j}\right)} / \hbar$, with $\mu$ being the atom-diatom reduced mass.

\section{Statistical quantum method}

The SQM developed by Manolopoulos and co-workers ${ }^{27,28}$ has been employed to study the dynamics of the title reaction. The method is conceived to treat insertion reactions which proceed via the formation of an intermediate complex. It has been largely used in the past and comparisons with both EQM and experimental results has proved it to be an adequate approach for complex-forming reactions. ${ }^{48}$ The method treats the formation and subsequent fragmentation of the intermediate species separately and the state-tostate reaction probability, at specific value of $J$ and the QM parity eigenvalue $I, P_{i f}^{I J}\left(E_{c}\right)$, is calculated as the product of the individual capture probability for the complex to be formed from the initial state $i$ and the fraction of collision complexes which decay to the final state $f$. In order to avoid previous deficiencies found at the state-to-state level ${ }^{21,29}$ for the $\mathrm{H}^{+}+\mathrm{H}_{2}$ reaction, we have employed in this study the CC version of the SQM, in which no CS approximation is assumed for calculating the corresponding capture probabilities in the reactant and product channels. The calculation includes explicitly both product $\mathrm{HD}+\mathrm{D}^{+}$arrangements.

The method only produces values for the state-to-state reaction probability [the square modulus of the scattering matrix $S^{J}(E)$ ] and therefore, the calculation of DCSs requires the application of the random phase approximation, as mentioned in Sec. II B, which neglects interference effects between different values of $J$ and $I$ in the exact expression of the DCS. ${ }^{28}$ The value of the capture radii defining the region in which the intermediate complex is assumed to be formed is $3 a_{0}$ in Jacobi mass-scaled coordinates for both arrangements, and all initial and final rovibrational states energetically open at $1.1 \mathrm{eV}$ total energy were considered in the calculation.

\section{Quasiclassical trajectory method}

QCT calculations have been performed for the title reaction at the two fixed collision energies of 0.1 and $0.524 \mathrm{eV}$ by running batches of $2 \times 10^{5}$ trajectories at each energy following the procedures described in detail elsewhere. ${ }^{49}$

The integration step size in the trajectories was chosen to be $5 \times 10^{-17} \mathrm{~s}$. This guarantees a total energy conservation better than one part in $10^{4}$ and conservation of total angular momentum better than one part in $10^{6}$. The trajectories were started at a distance between the incoming atom and the center of mass of the diatomic of $17 \AA$, given the long-range interaction in the entrance channel of the potential surface.

The rovibrational energies of the HD product molecules were calculated by semiclassical quantization of the action 
using the potential given by the asymptotic diatom limits of the PES. These rovibrational energies were fitted to Dunham expansions containing 20 terms [fourth power in $v+1 / 2$ and third power in $j(j+1)]$. The assignment of product quantum numbers $\left(v^{\prime}, j^{\prime}\right)$ was carried out by equating the classical rotational angular momentum of the product molecule to $\left[j^{\prime}\left(j^{\prime}+1\right)\right]^{1 / 2} \hbar$. With the (real) $j^{\prime}$ value so obtained, the vibrational quantum number $v^{\prime}$ was found by equating the internal energy of the outgoing molecule to the corresponding Dunham expansion. In the most common procedure, these real $v^{\prime}$ and $j^{\prime}$ values are rounded to the nearest integer, in what is named the histogramatic binning method. As in previous works, ${ }^{50,51}$ we have used a Gaussian-weighted binning $(\mathrm{GWB})^{52}$ procedure, in which a Gaussian function centered at the quantal action and with a given width has been used to weigh the trajectories following the criteria that the closer the vibrational action of a given trajectory to the nearest integer, the larger the weighting coefficient for that trajectory. In particular, in the present work we have used a full width at half maximum for the Gaussian functions of 0.1 at $0.524 \mathrm{eV}$ collision energy, whereas a value of 0.02 had to be used at $0.1 \mathrm{eV}$ collision energy for a better agreement with the EQM results.

Using the GWB procedure, DCSs were calculated at the two collision energies for every rovibrational state of the HD product molecule by the method of moments expansion in Legendre polynomials. The Smirnov-Kolmogorov test was used to decide when to truncate the series. Significance levels higher than $99 \%$ could be achieved by using eight to 16 moments, depending on the number of reactive trajectories available, ensuring good convergence, such that the inclusion of more terms did not produce any significant change.

The collision energy evolution of the reaction probability at different values of the total angular momentum $J=0,10$, 20,30 , and $40, P^{J}\left(E_{c}\right)$, for the title reaction have been calculated by running batches of $10^{5}$ trajectories for each value of $J$ in the collision energy range $0.005-1.6 \mathrm{eV}$ as described in Ref. 49 using the expression

$$
b=\frac{\hbar}{\mu v_{r}}[J(J+1)]^{1 / 2},
$$

where $b$ is the impact parameter of the trajectories and $\mu$ and $v_{r}$ are the $\mathrm{HD}_{2}^{+}$reduced mass and relative velocity, respectively. The calculation of the vibrationally state-resolved reaction probabilities for $J=0$ has been performed by the method of moments expansion in Legendre polynomials and employing the GWB procedure commented on above to assign final vibrational states. The integration step size and the initial distance between the incoming atom and the center of mass of the diatomic were the same as in the batches at fixed collision energy mentioned above.

\section{EXPERIMENT}

\section{A. Apparatus}

The measurements presented in this paper correspond to a previously reported study ${ }^{2}$ and only a brief outline of the experiment will be given here. The experiment was carried out using the apparatus described in detail in Ref. 53 modi- fied to measure the state-resolved reaction of Rydberg $\mathrm{H}$ atoms (denoted $\mathrm{H}^{*}$ ) with deuterium molecules: $\mathrm{H}^{*}+\mathrm{D}_{2}$ $\rightarrow \mathrm{HD}+\mathrm{D}^{*}$. As already mentioned in the Introduction section, this experiment and a similar study have recently shown that this reaction with Rydberg atoms is equivalent to the corresponding $\mathrm{H}^{+}+\mathrm{D}_{2} \rightarrow \mathrm{HD}+\mathrm{D}^{+}$ion-molecule reaction. ${ }^{1-3}$ This similarity has its origin in the large and slow orbit of the Rydberg electron which acts as a spectator while the ionmolecule reaction takes place. Additionally, the Rydberg electron shields the charge of the ion so that the ion-molecule reaction can be measured without adverse space-charge effects found in ion-beam experiments.

The experiment followed a crossed beams approach where Rydberg $\mathrm{H}^{*}$ atoms collide at right angle with a pulsed molecular beam of para- $\mathrm{D}_{2}$ adiabatically cooled to the ground rotational state $(j=0)$. Fast $\mathrm{H}$ atoms were generated from the photodissociation of $\mathrm{HI}$ in a second pulsed molecular beam (parallel to the $\mathrm{D}_{2}$ beam) with linearly polarized $266 \mathrm{~nm}$ light. Prior to collision, the $\mathrm{H}$ atoms were excited into the $n=36$ Rydberg level by resonant two photon excitation using laser light of 121.6 and $365.8 \mathrm{~nm}$. The experimental collision energy was determined by a Monte Carlo simulation of the experiment (see below) to be $E_{c}$ $=0.529 \pm 0.002 \mathrm{eV}$. Both reactively and nonreactively scattered Rydberg atoms, $\mathrm{D}^{*}$ and $\mathrm{H}^{*}$, respectively, were detected using a particle detector rotatable in the plane of the molecular beams. Note that the detection method was only sensitive to Rydberg atoms (for details see Ref. 2). The time-of-flight (TOF) spectra of the Rydberg atoms were measured at different laboratory scattering angles $\left(0^{\circ}\right.$ corresponds to the direction of the incident $\mathrm{H}^{*}$ beam) and were converted into KES in the laboratory frame.

\section{B. Simulation of laboratory kinetic energy spectra}

In order to assess the performance of the different theoretical DCSs against the experimental data, a Monte Carlo simulation of the experiment was performed using each set of DCSs as an input. As only reactive DCSs are available only reactive collisions were simulated (see discussion of the spectra below). The simulation follows the paths of the HI molecule from the nozzle to the dissociation point, of the $\mathrm{H}$ atom from the dissociation to the collision with the deuterium molecule and of the product $\mathrm{D}^{*}$ atom from the collision to the detector. The parameters of the simulation are the size, densities, and speed distributions of the molecular beams, the pulse intensities (beam and temporal profiles) and linewidths of the laser beams, the relative timing between the dissociation and excitation laser pulses, and the geometry and the position of the detector. A key aspect of the simulation is the Rydberg excitation of the $\mathrm{H}$ atoms. A 3-level population dynamics calculation is performed for each $\mathrm{H}$ atom to evaluate the probability of excitation to a Rydberg level. The relative populations of the $1 s, 2 p$, and $36 d$ levels are evaluated by factoring the Einstein coefficients and the laser field intensity experienced by each atom as it travels in and out of the excitation lasers' paths. In practice, the excitation takes place in a small electric field which mixes the $l$ and $m_{l}$ sublevels of the Rydberg state leading to long lived Rydberg atoms. Our 
simulation neglects the detailed dynamics of the $l$ mixing process. This is justified by the very long lifetime of the $36 d$ level which is dominated by the spontaneous decay to the $2 p$ level. With respect to the simulation, the $36 d$ population does not significantly change during the flight of the $\mathrm{H}^{*}$ atom from the excitation to the collision point.

For each collision the Newton diagram is constructed from which the laboratory speed and consequently the TOF of the product $\mathrm{D}^{*}$ Rydberg atom to the detector is determined. The Monte Carlo simulation builds up each TOF spectrum by taking into account all the common weighting factors describing the effects of the molecular beams, the laser beam intensities, and the geometry of the experiment as listed above. For each product quantum state, the theoretical DCS is transformed from the center of mass (CM) into the laboratory frame using the corresponding Jacobi factor and used as the final weighting factor when adding the contribution of the individual collision to the TOF spectrum. Finally, the simulated TOF spectra are converted into laboratory KES and compared to the experimental spectra as shown below.

Recently, Hayes and Skodje performed a theoretical study of the dynamics of the Rydberg electron in the $\mathrm{H}^{*}$ $+\mathrm{D}_{2} \rightarrow \mathrm{HD}+\mathrm{D}^{*}$ reaction ${ }^{4}$ in order to investigate deviations of the quasiclassical DCSs from the experimental results by Song et al. ${ }^{3}$ They examined the effect of (i) the impulse imparted on the Rydberg electron by the ion-molecule collision, (ii) the deexcitation of the Rydberg electron by spontaneous emission, and (iii) the ionization of the Rydberg electron by the small electric field present in the experiment. All three effects lead to a small, scattering angle dependent attenuation of the Rydberg atom signal in the TOF measurements. In the context of the simulations presented here, these attenuations are negligible, in particular, because the CM scattering angles do not change significantly in each KES.

However, the change of momentum of the ion due to the (reactive) collision will change the motion of the frame of reference for the Rydberg electron. Thus, the impulse imparted via the Coulomb interaction onto the Rydberg electron can change its Rydberg state, leading to a distribution of product Rydberg levels. ${ }^{4}$ This change of the energy of the Rydberg electron will lead to an additional spread of the products' kinetic energies which is not accounted for by our simulations. Indeed, the peaks in our simulated KES are noticeably narrower than found experimentally.

In order to facilitate a better comparison between theory and experiment each KES is simulated as follows: (i) the experimental energy scale is stretched and shifted to account for uncertainties in the exact length of the flight paths; (ii) each peak in a KES is simulated by a Lorentzian profile with the area determined by the Monte Carlo simulation but using the experimental peak width.

\section{RESULTS}

In this section we present the results of our study on the title reaction. We start with an analysis of the reaction probabilities as a function of collision energy obtained by means of the theoretical methods described in Sec. II. Total and vibrationally resolved probabilities at zero total angular mo-

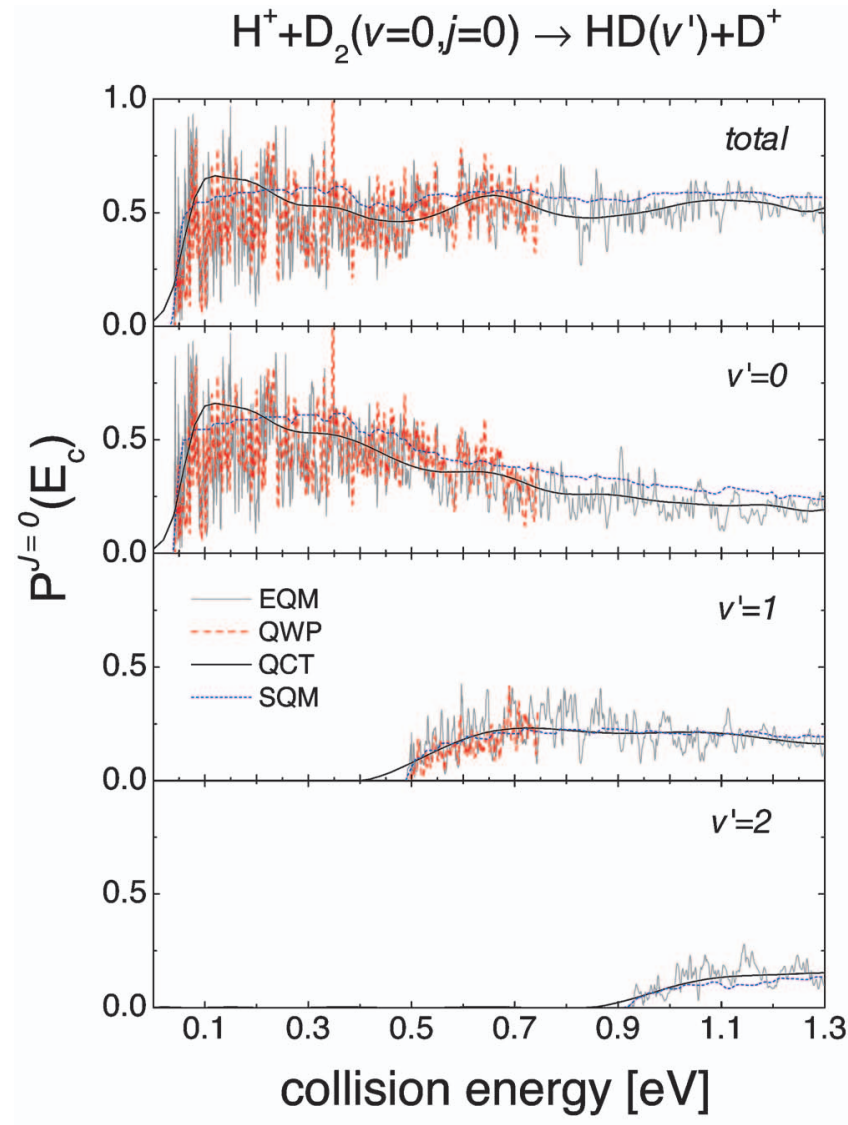

FIG. 1. (Color) Total and vibrationally state resolved reaction probabilities as a function of collision energy, at zero total angular momentum, $P^{J=0}\left(E_{c}\right)$, for the $\mathrm{H}^{+}+\mathrm{D}_{2}(v=0, j=0) \rightarrow \mathrm{HD}\left(v^{\prime}\right)+\mathrm{D}^{+}$reaction calculated by means of the EQM (gray solid line), QWP (red dashed line), SQM (blue dotted line), and QCT (solid line) approaches.

mentum $(J=0)$ are completed with the $J=10,20,30$, and 40 cases. We also include a particularly detailed investigation of the dynamics of the $\mathrm{H}^{+}+\mathrm{D}_{2}(v=0, j=0) \rightarrow \mathrm{HD}+\mathrm{D}^{+}$reaction at $0.524 \mathrm{eV}$ collision energy. Besides the total reaction probability, calculations of the corresponding ICSs and DCSs at that specific collision energy have been performed. The theoretical predictions are extensively compared with existing experimental work on the reaction. In particular, we present comparisons of simulated KES with those obtained in the experiment. ${ }^{2}$ The analysis is finally extended to the lower collision energy $E_{c}=0.1 \mathrm{eV}$, in order to investigate if possible changes in the main reaction mechanism of the title reaction are appreciated.

\section{A. Reaction probabilities as a function of collision energy}

The total and vibrationally resolved reaction probabilities for $J=0$ calculated up to $1.3 \mathrm{eV}$ collision energy are shown in Fig. 1. Results obtained by means of the EQM, QWP, SQM, and QCT approaches are compared. The EQM and QWP probabilities exhibit very dense large amplitude oscillations related with the existence of a large number of resonant states, which, as in the case of the corresponding $\mathrm{H}^{+}+\mathrm{H}_{2}$ isotopic variant, are due to the deep potential well. In fact, we only present the QWP result up to $E_{c}=0.75 \mathrm{eV}$, in 
order to facilitate the pictorial comparison in the figure. There is no full agreement in the precise position of the numerous resonance peaks in the two sets of data. In this regard, it is worth mentioning that the precise location of each resonance, some of them corresponding to very high excitations, may depend on the coordinate representation employed in the calculation, complicating therefore the achievement of a similar degree of convergence. Nevertheless, both exact quantal results show a very similar behavior and clearly indicate a process mediated by very narrow resonances.

This dynamical scenario is consistent with the fairly good average description provided by the SQM and QCT approaches within the energy range under study. The present result confirms previous findings for ion-diatom reactions between different isotopes of $\mathrm{H}$, in which reaction probabilities for $J=0$ have been successfully reproduced by statistical procedures. ${ }^{13,16,20,21}$ The QCT results seem to differ slightly only from such an average agreement with the exact probabilities in the thresholds for the different processes shown in Fig. 1. Apart from that, the classical probability, specially in the case of reactions leading to $\operatorname{HD}\left(v^{\prime}=1,2\right)$ fragments (bottom panels of Fig. 1), is almost indistinguishable from the SQM prediction.

Differences among the theoretical predictions start to manifest when we focus on the reaction at $J>0$. As shown in Fig. 2, the comparison of the QWP, QCT, and SQM reaction probabilities at $J=10,20,30$, and 40 reveals that both QWP and QCT approaches predict a significant faster reduction on the total reactivity when the total angular momentum increases with respect to the SQM result. Thus, despite the good agreement found at $J=10$ between the three methods, both QWP and SQM yield sensibly different results at the larger total angular momenta shown in the figure. In fact, the QCT probabilities, smaller in all cases than the SQM, seem to be in a better overall average agreement with the QWP result. Moreover, the classical reaction thresholds for the $J$ $>0$ cases are in better agreement with the corresponding quantal values than at $J=0$. These discrepancies between the QWP and SQM approaches were first observed in previous studies on both the title ${ }^{20}$ and $\mathrm{H}^{+}+\mathrm{H}_{2}$ (Ref. 21) reactions. Interestingly, as shown in Fig. 2, the EQM reaction probabilities calculated in the vicinity of $E_{c}=0.524 \mathrm{eV}$ at $J=40$ (see inset) are in good general accord with the statistical results and, thus, we can conclude that the SQM approach is giving an acceptable picture of the reaction at such large values of $J$.

\section{B. Dynamics at $0.524 \mathrm{eV}$ collision energy \\ 1. Opacity function}

One of the collision energies which has been investigated in detail in the present work is $E_{c}=0.524 \mathrm{eV}$. Figure 3 shows the reaction probability as a function of the total angular momentum $J$, i.e., the opacity function, calculated at this specific collision energy by means of the EQM, QWP, QCT, and SQM approaches.

An interesting feature manifested in the figure is that at low $J$ values $(J \leqslant 20)$, all methods predict reaction probabili-
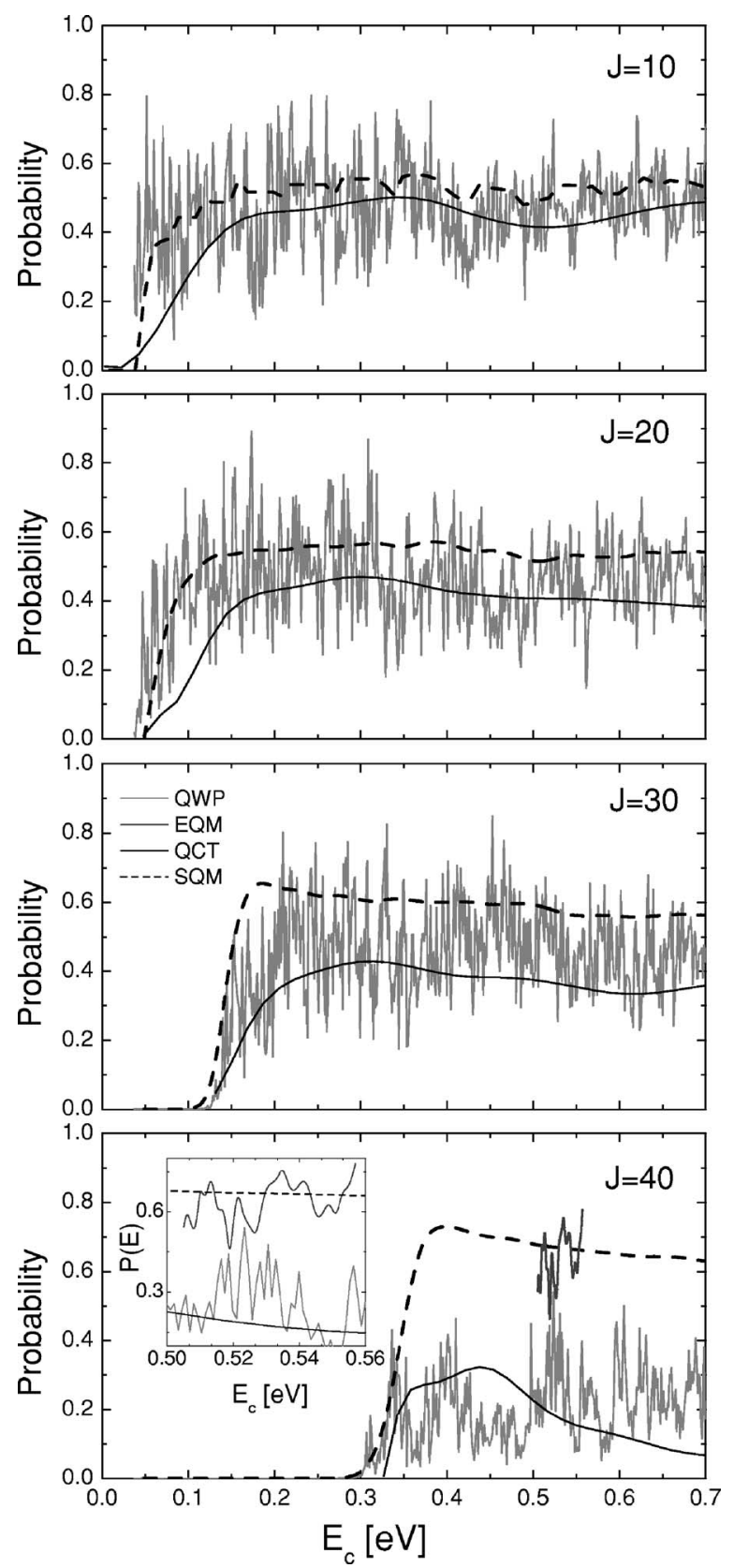

FIG. 2. Total reaction probabilities as a function of collision energy, at $J$ $=10,20,30$, and 40 , for the $\mathrm{H}^{+}+\mathrm{D}_{2}(v=0, j=0) \rightarrow \mathrm{HD}\left(v^{\prime}\right)+\mathrm{D}^{+}$reaction calculated by means of the QWP (grey solid line), SQM (dashed line), and QCT (solid line) approaches. For $J=40$, the inset shows the comparison of these three methods with the EQM probabilities (dark gray line) calculated around $0.524 \mathrm{eV}$ collision energy.

ties in an overall good agreement. Both EQM and QWP results exhibit an oscillatory behavior in terms of $J$, as a result of the large number of existing resonances in the reaction, which seems to be even in phase for the lowest values of $J(J<10)$. The QCT and SQM approaches provide an average description of the exact and wave packet probabilities in this range of $J$ 's.

The comparison between the EQM and QWP probabilities resembles some of the previous findings for the $\mathrm{H}^{+}$ $+\mathrm{H}_{2}$ reaction. ${ }^{21}$ There is also in this case a sudden fall of the QWP probabilities beyond $J \sim 35$. In fact, the disagreement with the EQM calculations for $J=40$ around the vicinity of 


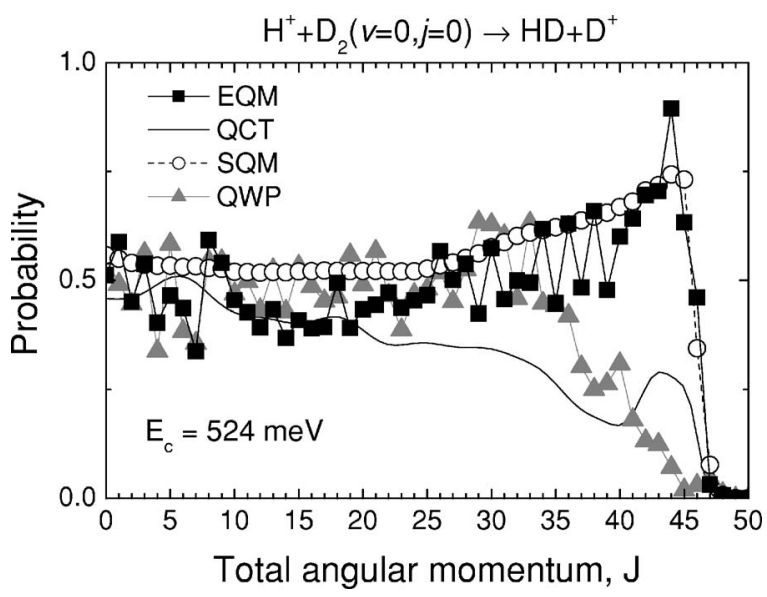

FIG. 3. Opacity function or reaction probability in terms of the total angular momentum $J$ calculated at $0.524 \mathrm{eV}$ collision energy for the $\mathrm{H}^{+}+\mathrm{D}_{2}(v$ $=0, j=0$ ) reaction by means of the EQM (full squares with solid line), QWP (gray full triangles and solid line), SQM (empty circles and dashed line), and QCT (solid line) approaches.

$E_{c}=0.524 \mathrm{eV}$ shown in the bottom panel of Fig. 2 indicates that the discrepancies manifested at large values of $J$ displayed in Fig. 3 are not accidental. Since the QWP approach gives account for the correct energy threshold, the rotational barrier for the initial $\mathrm{D}_{2}(v=0, j=0)$ state seems to be reasonably well described. Therefore, the disagreement might have its origin in the exit of the products after the complex is formed. It seems that the present QWP approach is not able to reproduce appropriately the density of resonances effectively coupled to the $\operatorname{HD}\left(v^{\prime}, j^{\prime}\right)$ product states. Such failure of the QWP approach is attributed to the strong limitation introduced by considering a single helicity component, which is noticeable, at least at $E_{c}=0.524 \mathrm{eV}$, for $J>35$.

The description of the reaction probability provided by the SQM approach is reasonably good in comparison with the EQM result. The statistical prediction correctly describes the behavior of the EQM opacity function at the final values of $J$. The agreement between both approaches at this collision energy is consistent with the situation shown in Fig. 2, where the SQM method describes on average the EQM reaction probabilities at $J=40$ within the energy range around $E_{c}=0.524 \mathrm{eV}$. It is true nevertheless that the statistical results remain slightly over the exact probabilities, agreeing mainly with the maximum peaks of the oscillations in the EQM opacity function. This certainly differs with the corresponding comparison carried out for the $\mathrm{H}^{+}+\mathrm{H}_{2}$ reaction, where the SQM probability curve was perfectly describing, on average, the oscillating EQM results (see Fig. 10 in Ref. 21).

At $J \sim 20$ the QCT curve starts to underestimate the EQM probabilities. This feature, which was also observed for the $\mathrm{H}^{+}+\mathrm{H}_{2}(v=0, j=0)$ reaction at $E_{c}=0.44 \mathrm{eV},{ }^{21}$ seems to be related to the existence of a large number of trajectories which despite to reach the region of the potential well and to be trapped for some finite time $(\tau \sim 500 \mathrm{fs})$ are, however, directed back to the reactant arrangement. Moreover, the number of such trajectories increases with $J$. In other words, the intermediate complex gains a considerable amount of rotational energy to finally fragment again into the reactants. In this sense, the failure of both the QCT and QWP ap-

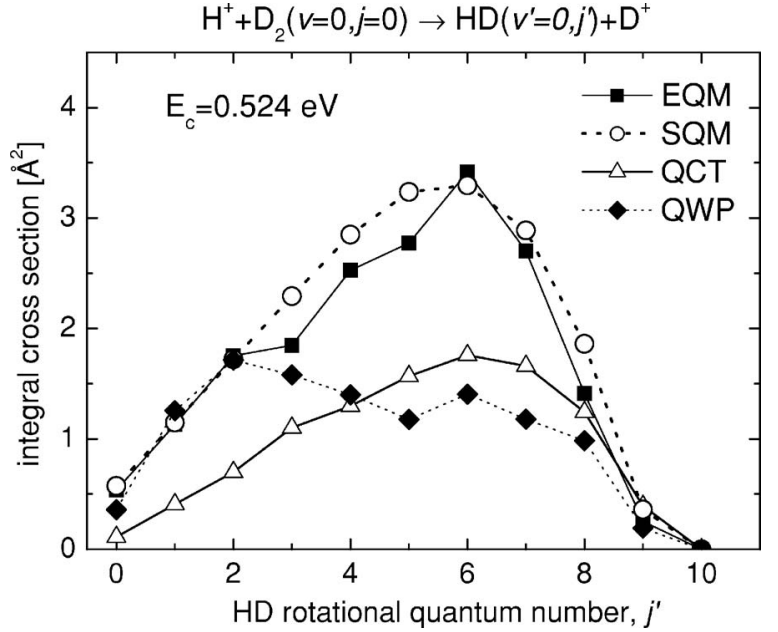

FIG. 4. Integral cross sections (in $\AA^{2}$ ) calculated at $E_{c}=0.524 \mathrm{eV}$ for the $\mathrm{H}^{+}+\mathrm{D}_{2}(v=0, j=0) \rightarrow \mathrm{HD}\left(v^{\prime}=0, j^{\prime}\right)+\mathrm{D}^{+}$reaction by means of the EQM (full squares and solid line), QWP (solid diamonds and dotted line), SQM (empty circles and dashed line), and QCT (empty triangles and solid line) methodologies.

proaches to provide the correct opacity function could be connected to deficiencies to describe the precise way trajectories and wave packets abandon the potential well region once they get trapped inside. On the other hand, an estimation of the reaction probability by using exclusively the reactive trajectories which end in the product fragments after spending a considerable period of time in the potential well region yields an opacity function in close similarity to the SQM prediction. In this sense, the SQCT approach ${ }^{29,30}$ produces reaction probabilities in perfect accord with the present SQM results, which indicates that the observed deficiencies of the QCT approach should not be attributed only to the existence of purely QM effects.

\section{Integral cross sections}

Product rotational distributions have been calculated at this collision energy. Figure 4 shows the comparison among the final rotationally state-resolved ICSs for the title reaction as predicted by the different theoretical approaches used in the present work. The reaction channel for the formation of vibrationally excited $\operatorname{HD}\left(v^{\prime}=1\right)$ is accessible at this collision energy, but only rotational states up to $j^{\prime}=2$ are populated. As can be seen, the $\mathrm{EQM} \mathrm{HD}\left(v^{\prime}=0\right)$ rotational distribution shows a closely statistical profile, with a monotonical increase in the population of the $\operatorname{HD}\left(v^{\prime}=0, j^{\prime}\right)$ states, reaching a maximum value at $j^{\prime}=6$ and then decreasing to finally end at $j^{\prime}=10$. It is not surprising then that the SQM calculation provides a reasonably good counterpart for the EQM rotational distribution. Interestingly, the EQM cross section does not exhibit the markedly oscillatory behavior with $j^{\prime}$ found for the $\mathrm{H}^{+}+\mathrm{H}_{2}$ reaction. ${ }^{21}$ The QCT ICSs, which are clearly below the EQM and SQM ones, do not match any of the EQM values for $j^{\prime}<8$ states. In this sense, a scaling of the QCT results to the EQM ones shows that the shape of the QCT distribution is not very far from those of the EQM and SQM. The QWP rotational distribution manifests a nearly bimodal behavior, with maxima at $j^{\prime}=2$ and $j^{\prime}=6$. The 
TABLE I. Total and vibrationally state selected integral cross sections (in $\left.\AA^{2}\right)$ calculated at $0.524 \mathrm{eV}$ for the $\mathrm{H}^{+}+\mathrm{D}_{2}(v=0, j=0)$ reaction using the different theoretical methods employed in the present work, i.e., EQM, QWP, SQM, and QCT.

\begin{tabular}{lrrrr}
\hline \hline & EQM & QWP & SQM & QCT \\
\hline$\sigma_{\text {tot }}$ & 18.54 & 11.63 & 20.45 & 10.43 \\
$\sigma_{v^{\prime}=0}$ & 18.35 & 11.25 & 20.23 & 10.24 \\
$\sigma_{v^{\prime}=1}$ & 0.19 & 0.38 & 0.22 & 0.19 \\
\hline \hline
\end{tabular}

agreement with the EQM distribution up to $j^{\prime}=2$ is perfect, but the trend in the rest of rotational states clearly disagrees. The above mentioned possible deficiencies to describe outgoing wave packets from the potential well region to the products arrangement can be also affecting specially to those cases in which the HD diatom is formed in high rotational states. For such situations, high values of $\Omega$ which are not included within the ACS approximation of the QWP calculation are perhaps required.

The comparison is extended, in Table I, to the total and vibrationally state-resolved ICSs. These results confirm that reaction proceeds mainly by forming vibrationless $\mathrm{HD}$, and that the EQM ICSs are partially reproduced by the SQM method. On the other hand, both QWP and QCT results remain well below the EQM calculations.

\section{Differential cross sections}

As indicated in Sec. I, one of the main incentives of considering the precise collision energy of $0.524 \mathrm{eV}$ for a detailed theoretical investigation of the dynamics of the title reaction is the existence of substantial experimental results to compare with. In particular, one of the dynamical attributes which has received a preferential attention in the experiments by Song et $\mathrm{al}^{3}{ }^{3}$ are the DCSs. Angular distributions in the $\mathrm{CM}$ frame for the $\mathrm{H}^{+}+\mathrm{D}_{2}(v=0, j=0)$ reaction were reported, even at the state-to-state level, although in a limited angular range $\left(20^{\circ}<\theta<140^{\circ}\right)$.

In Fig. 5, the calculated total DCS for the title reaction are compared with the measurements reported in Ref. 3. For this comparison, the experimental value has been scaled to match with the EQM cross section at the sideways scattering direction $\left(\theta \approx 90^{\circ}\right)$. The agreement between EQM and experiment is reasonably good, although the experimental DCS seems to be somewhat more asymmetric than the EQM one. Given this choice for scaling between theory and experiment, the overall agreement around the sideways scattering direction found between the EQM and SQM DCSs enables the statistical result to provide a good description of the measured results. The QCT and QWP angular cross sections, however, remain below the experimental values for this range, although the shape of the DCSs is very similar in the angular range where there is experimental data. This is more clearly appreciated in the inset of Fig. 5, where theoretical and experimental DCSs are compared for $20^{\circ} \leqslant \theta \leqslant 140^{\circ}$, the angular range for which the measured values are restricted.

No information regarding scattering at the forward and backward directions is obtained from the experiment. It is,

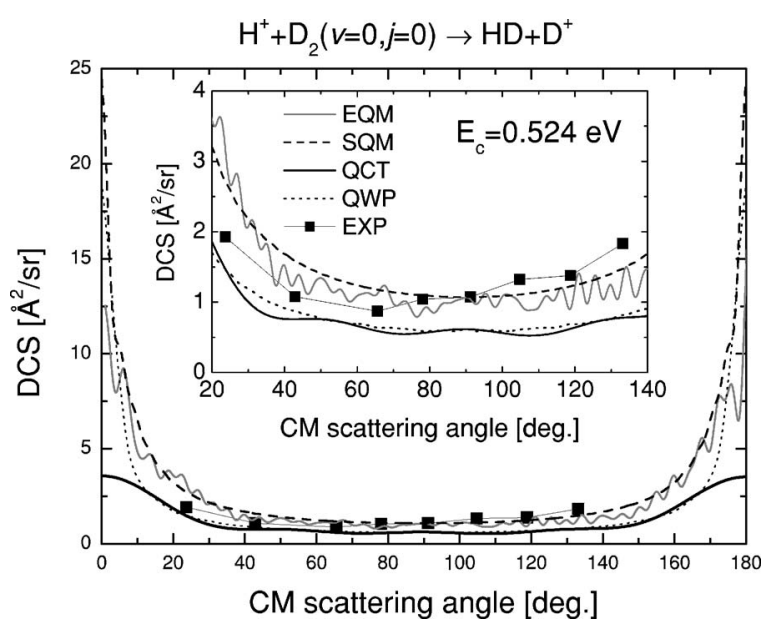

FIG. 5. Total differential cross section (in $\AA^{2} / \mathrm{sr}$ ) calculated at $0.524 \mathrm{eV}$ collision energy for the $\mathrm{H}^{+}+\mathrm{D}_{2}(v=0, j=0)$ reaction using the EQM (gray solid line), QWP (dotted line), SQM (dashed line), and QCT (solid line) methods. The theoretical DCSs are compared with the experimental results of Ref. 3, which are shown in full squares and solid line. The experimental results, restricted to the angular range $20^{\circ}<\theta<140^{\circ}$, have been scaled to the EQM DCS at $\theta=90^{\circ}$. The inset shows a magnification of the angular range between $20^{\circ}$ and $140^{\circ}$.

however, around these particular angular directions where the different theoretical calculations differ at most. The EQM DCS is only slightly asymmetric with respect to the sideways direction, with values of $\sim 12.5$ and $\sim 18.5 \AA^{2} /$ sr at forward and backward, respectively. One of the most striking features of the exact angular distribution is the significant differences found with respect to the DCS for the $\mathrm{H}^{+}+\mathrm{H}_{2}$ reaction at $E_{c}=0.44 \mathrm{eV}^{21}$ In that case, the EQM DCS shows a more marked polarization, with forward-backward peaks about 30 times more intense than the values found at the sideways direction. This ratio is clearly smaller in the present case. Interestingly, the SQM method does not describe entirely well the EQM forward and backward peaks. In fact, the equally probable DCS obtained by means of the SQM at those two directions is significantly larger than the exact result $\left(\sim 24.5 \AA^{2} / \mathrm{sr}\right)$. In view of the final-rotational-stateresolved ICSs examined before (see Fig. 4), this extra cross section originated in the SQM predictions for the $j^{\prime}$ $=3-5,7,8$ states.

The QWP DCS, which also exhibits the forwardbackward symmetry enforced by the random phase approximation adopted in Eq. (1), shows a marked preference for the scattering at the $\theta=0^{\circ}$ and $\theta=180^{\circ}$ directions, but clearly improves the SQM result with respect to EQM, yielding a value of $\sim 18.2 \AA^{2} /$ sr for both peaks, in good agreement with the EQM result at the backward direction. This suggests that the QWP calculation describes well low values of $j^{\prime}$ and $\Omega=0$ (but no high $j^{\prime}$ and $\Omega$ ). The QCT calculation, as in the case of the $\mathrm{H}^{+}+\mathrm{H}_{2}$ reaction, ${ }^{21}$ fails to account for most of the forward and backward peaks in the DCS. As it has been discussed before, those peaks appear on the contrary in the SQCT result. ${ }^{29,30}$ The present QCT total DCS is similar ${ }^{54}$ to that shown in the work by Song et al., ${ }^{3}$ although the PES used was different. In that work, however, the QCT DCS is only shown at the limited angular range of the experiment.

DCSs have been further analyzed at a higher detail, and 


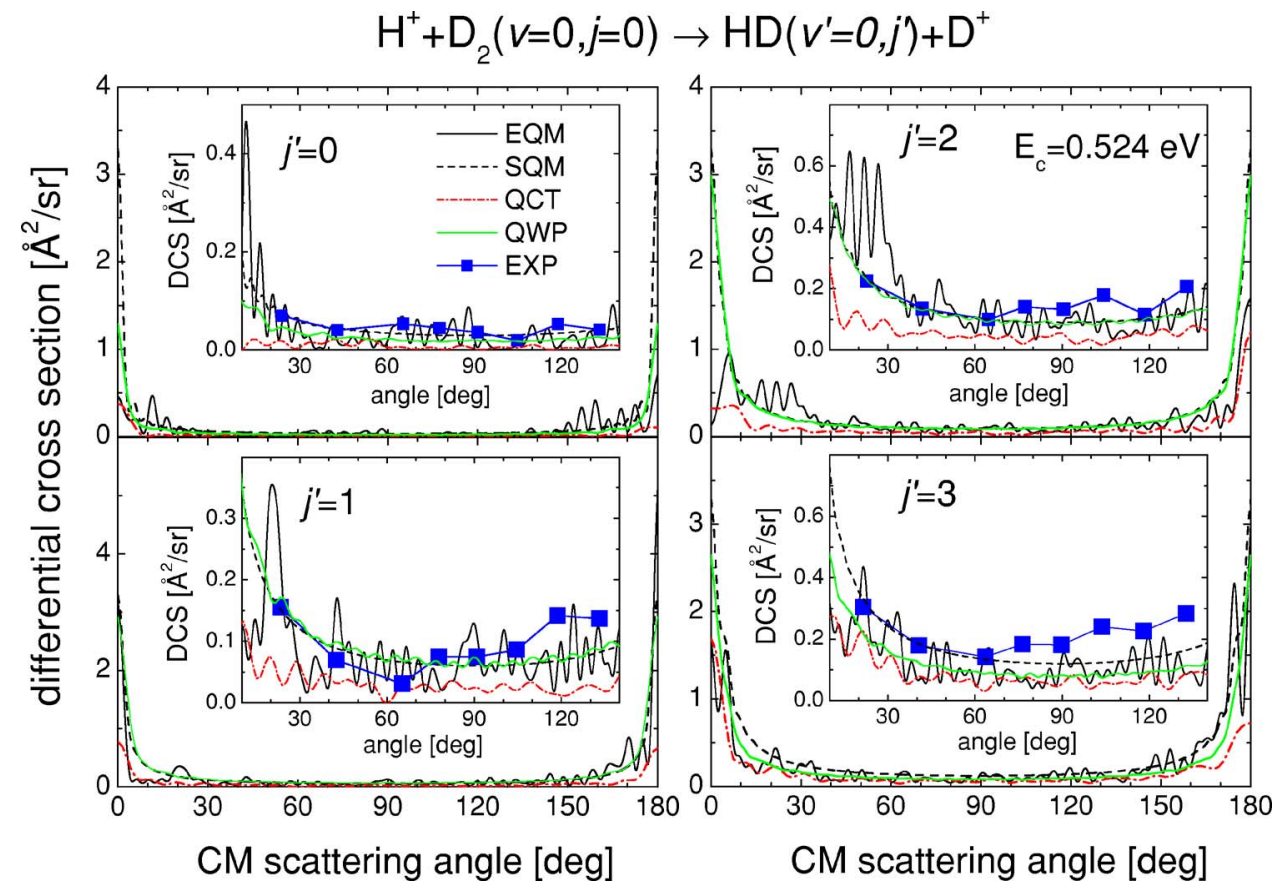

FIG. 6. (Color online) Rotationally state resolved differential cross sections (in $\AA^{2} / \mathrm{sr}$ ) calculated at $0.524 \mathrm{eV}$ collision energy for the $\quad \mathrm{H}^{+}+\mathrm{D}_{2}(v=0, j=0) \rightarrow \mathrm{HD}\left(v^{\prime}\right.$ $\left.=0, j^{\prime}=0-3\right)+\mathrm{D}^{+}$reactions by means of the EQM (dark solid line), QWP (light solid line), SQM (dashed line), and QCT (dotted-dashed line) methods. As in Fig. 5, the theoretical distributions are compared with the stateto-state experimental cross sections reported by Song et al. (Ref. 3) (shown in solid squares with solid line). The insets show a magnification of the angular range between $20^{\circ}$ and $140^{\circ}$.

state-to-state angular distributions have been calculated by means of the different theoretical methods employed in this work. Results of such calculations are shown in Fig. 6 and 7 for the $\operatorname{HD}\left(v^{\prime}=0, j^{\prime}=0-3\right)$ and $\operatorname{HD}\left(v^{\prime}=0, j^{\prime}=4-7\right)$ states, respectively. The comparison with the experimental DCSs of Song et al. ${ }^{3}$ are displayed at the insets of each panel, with the only exception of $j^{\prime}=7$ in Fig. 7, for which no measurements were reported. The experimental DCSs have been scaled to match the EQM result at the sideways direction for $j^{\prime}=0$ and the same scaling factor has been employed for the rest of final rotational states. With this scaling, the experimental DCSs are fairly well described by most of the calculated angular distributions, with possibly the only exception of the QCT and the QWP results, which are slightly below the exact DCSs.
The EQM DCSs are highly oscillatory in the entire angular range and exhibit, in general for all $j^{\prime}$ states, certain symmetry with respect to the sideways scattering direction. The degree of polarization, which measures the preference for the forward-backward directions with respect to $\theta \sim 90^{\circ}$, is relatively small, with possibly the only exception of $j^{\prime}$ $=1$, in which the backward peak indicates some preference for the $\theta \sim 180^{\circ}$ scattering direction. This result differs from our previous findings for the $\mathrm{H}^{+}+\mathrm{H}_{2}$ reaction for which DCSs for some specific transitions exhibit a dramatic preference for either the forward or backward direction (see Fig. 7 of Ref. 21). Thus, in the present case, the agreement between the QCT and EQM DCSs at the forward and backward directions at some low $j^{\prime}$ states (as, for example, $j^{\prime}=0$ and $j^{\prime}=3$ ) is fairly good. The overall comparison between the

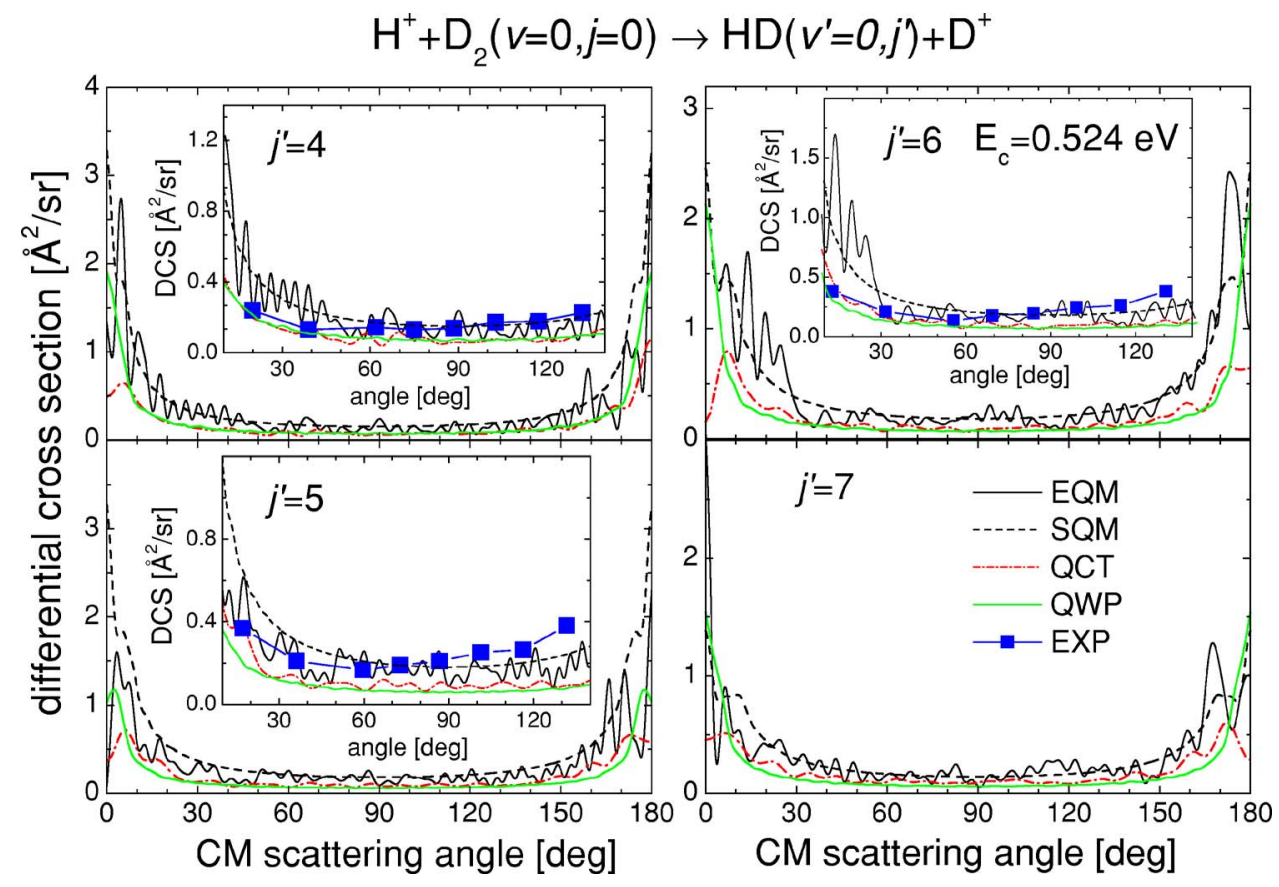

FIG. 7. (Color online) Same as Figure 6 but for the $\mathrm{H}^{+}+\mathrm{D}_{2}(v=0, j=0)$ $\rightarrow \mathrm{HD}\left(v^{\prime}=0, j^{\prime}=4-6\right)+\mathrm{D}^{+}$reactions. 
results obtained by means of both methods (EQM and QCT) reveals, nevertheless, that the QCT DCSs are somewhat below the EQM ones and that the agreement tends to improve as $j^{\prime}$ increases.

The evolution of the QWP DCSs as $j^{\prime}$ increases is quite interesting. Whereas an almost perfect agreement is found between the wave packet distributions and those obtained by statistical means at $j^{\prime}=1$ and $j^{\prime}=2$, the contribution at the sideways direction observed for the DCSs at larger $j^{\prime}$ clearly underestimates both exact and SQM predictions, approaching to the QCT result. As discussed above this might be an indication of the necessity of a larger number of helicity numbers in the calculation. At the forward and backward scattering directions, on the contrary, the QWP DCSs predict pronounced peaks, in a not bad agreement with the EQM values. The comparison carried out between the SQM and EQM DCSs reveals that although the SQM angular distributions provide, on average, a good description of the overall dynamics, the peaks at $\theta \sim 0^{\circ}$ and $\theta \sim 180^{\circ}$ in the EQM DCSs are not due to an entirely statistical process. The SQM yields more pronounced peaks at those angular regions, an overestimation which also extends along the sideways direction in some cases, as, for example, $j^{\prime}=3$ and $j^{\prime}=5$.

\section{Kinetic energy spectra}

The comparison between the experimental KES and the simulated ones based on the different theoretical DCSs is shown in Figs. 8 and 9. ${ }^{55}$ The experimental signal (arbitrary units) of each spectrum has been scaled to match roughly the $\mathrm{SQM}$ signal for the $\operatorname{HD}\left(v^{\prime}=0, j^{\prime}=6\right)$ quantum state. The scaling is partly a compromise since the $\mathrm{D}^{*}+\operatorname{HD}\left(v^{\prime}=0, j^{\prime}\right.$ =6) reactive signal may be slightly contaminated by $\mathrm{H}^{*}$ $+\mathrm{D}_{2}\left(v^{\prime}=1, j^{\prime}=4\right)$ inelastic signal. Note that the theoretical curves are not scaled with respect to each other.

In general, sideways scattering $\left(\Theta_{\mathrm{lab}}=52^{\circ}\right)$ is simulated better than backward scattering $\left(\Theta_{\text {lab }}=9^{\circ}\right)$. In particular, the low rotational product states are significantly overestimated by the theoretical DCSs in the $\Theta_{\mathrm{lab}}=9^{\circ}$ spectrum, which corresponds to an average $\mathrm{CM}$ scattering angle of $\theta_{\mathrm{CM}} \approx 172^{\circ}$. Presently, we do not have an explanation for this noticeable deviation between the experimental and simulated spectra at this scattering angle.

One might, in principle, say that the experimental spectra are best simulated using the SQM DCSs. The main deviation is seen for the high rotational product states $\left(j^{\prime}\right.$ $=7,8)$ where the SQM simulation underestimates the experimental signal in all the spectra. This may be due to the fact that the experimental collision energy was slightly higher than that of the theoretical studies. The overall performance of the QCT simulations is also good. The highly oscillatory nature of the EQM DCSs, particularly in the backward direction, can also be seen in the simulations. If the experimental spectra were rescaled so that parts of each spectrum fits the EQM simulation the remaining parts would be significantly over or underestimated. There seems not to be a clear trend regarding the EQM results.
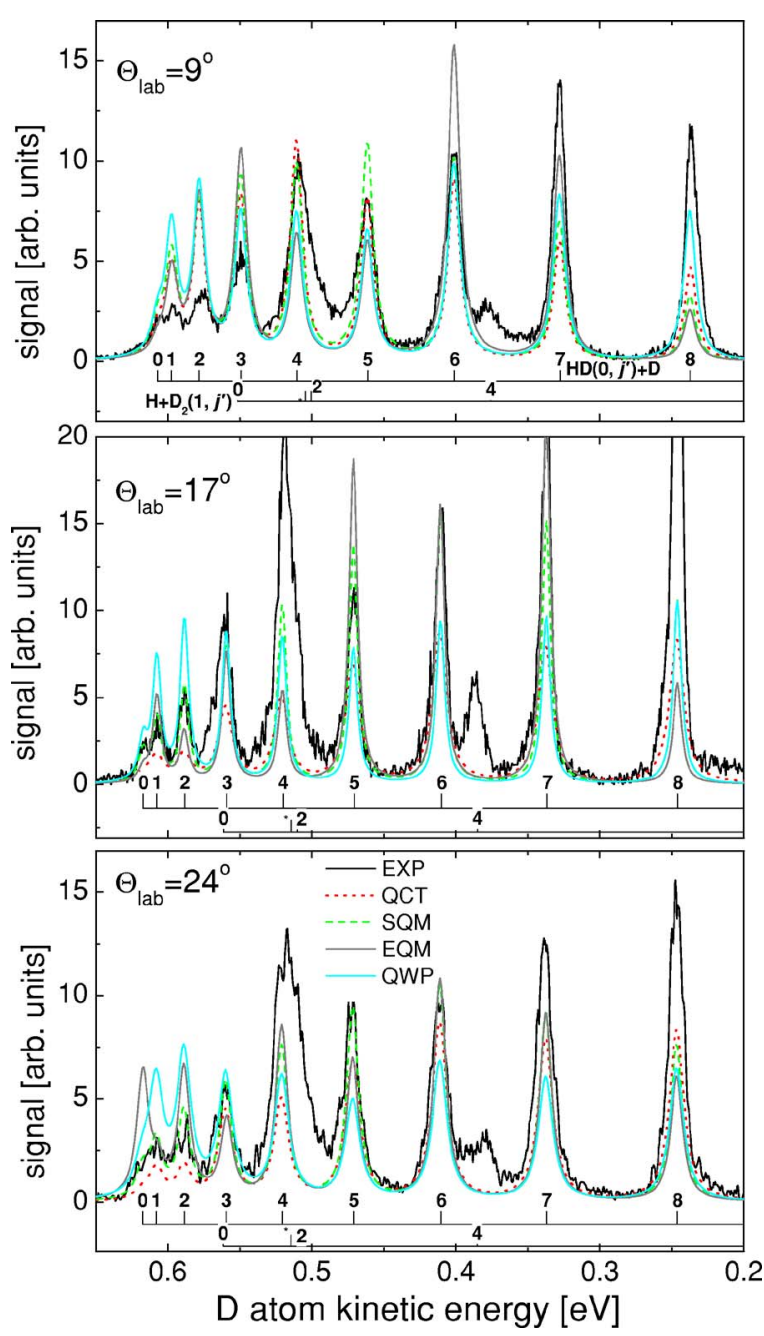

FIG. 8. (Color online) Kinetic energy spectra for the $\Theta_{\mathrm{lab}}=9^{\circ}, 17^{\circ}$, and $24^{\circ}$ laboratory angles at $0.524 \mathrm{eV}$ collision energy. Experimental results (dark solid line) and simulations obtained by using the EQM (solid line), SQM (dashed line), QCT (dotted line), and QWP (light solid line) results. The position of the different rotational states of reactively scattered $\operatorname{HD}\left(v^{\prime}\right.$ $\left.=0, j^{\prime}\right)$ and inelastically scattered $\mathrm{D}_{2}\left(v^{\prime}=1, j^{\prime}\right)$ are shown in the bottom of each panel.

\section{Dynamics at $0.1 \mathrm{eV}$ collision energy}

The title reaction has been theoretically studied at the lower collision energy $E_{c}=0.1 \mathrm{eV}$. These calculations are motivated by previous indications of distinct dynamics of the title reaction depending of the energy range considered. As discussed in Sec. I, the reaction seems to be governed by a complex forming mechanism at low collision energies to gradually shift to a more direct reaction pathway when collision energy increases. If this were the case, our present analysis at $E_{c}=0.1 \mathrm{eV}$ could reveal characteristic features in comparison with the results obtained at the larger collision energy of $0.524 \mathrm{eV}$.

The opacity function calculated at $E_{c}=0.1 \mathrm{eV}$ is shown in Fig. 10. The oscillations found at $E_{c}=0.524 \mathrm{eV}$ in the EQM results are also evident now, and seem even to be magnified at some ranges of large values of $J$ (as $10<J$ $<19$ and $20<J<27)$. At this collision energy, the QCT opacity function manages to describe the average behavior of the EQM one at a larger extent than in the case of $E_{c}$ 


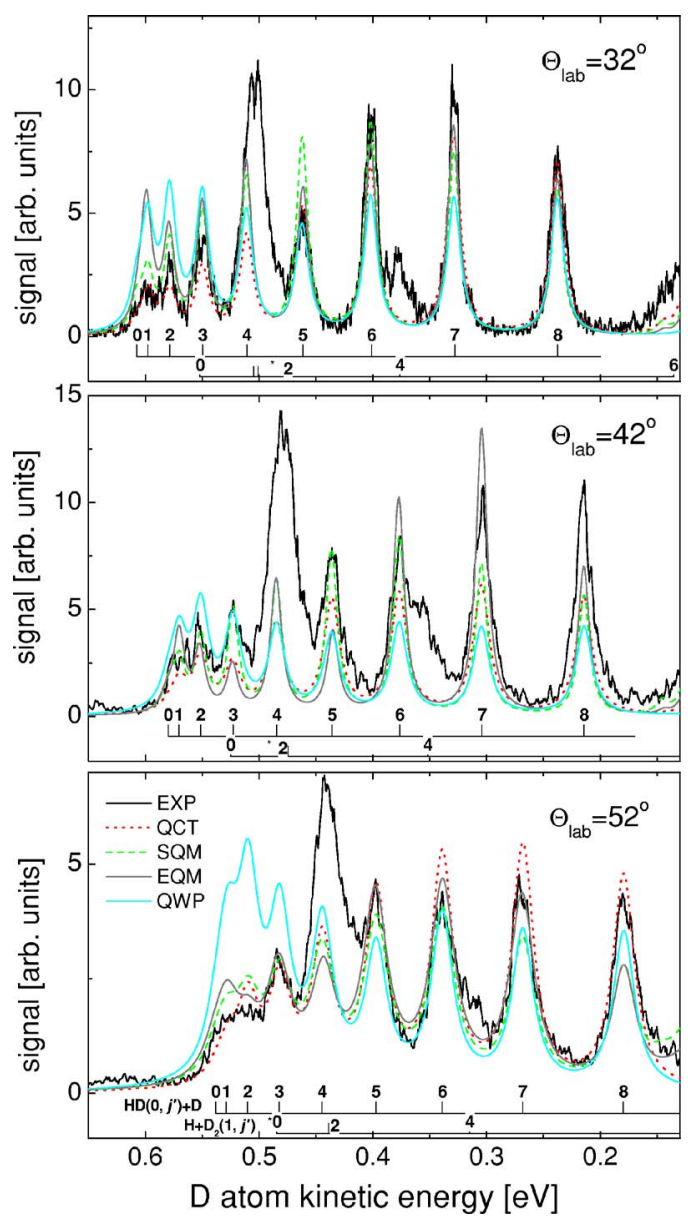

FIG. 9. (Color online) Same as Fig. 8 but for $\Theta_{\text {lab }}=32^{\circ}, 42^{\circ}$, and $52^{\circ}$.

$=0.524 \mathrm{eV}$. The QCT approach correctly reproduces the EQM opacity function with the only exception of a limited number of $J$ values. Analogously, the QWP opacity function does not show a sudden deviation with respect to the EQM one beyond a particular $J$ values. Besides, the oscillations are

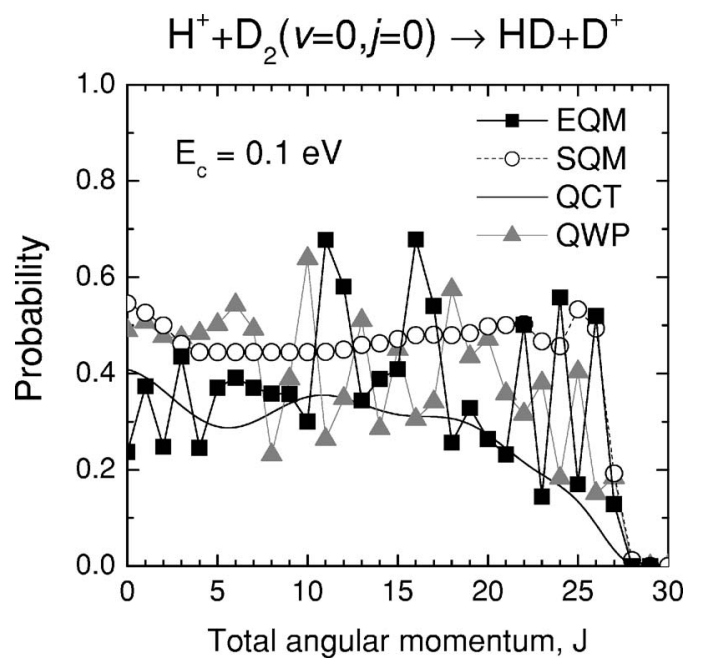

FIG. 10. Opacity function or reaction probability in terms of the total angular momentum $J$ calculated at $0.1 \mathrm{eV}$ collision energy for the $\mathrm{H}^{+}+\mathrm{D}_{2}(v$ $=0, j=0$ ) reaction by means of the EQM (full squares with solid line), QWP (gray full triangles and solid line), SQM (empty circles and dashed line), and QCT (solid line) approaches.
TABLE II. Total and vibrationally state selected integral cross sections (in $\left.\AA^{2}\right)$ calculated at $0.1 \mathrm{eV}$ for the $\mathrm{H}^{+}+\mathrm{D}_{2}(v=0, j=0)$ reaction using the different theoretical methods employed in the present work, i.e., EQM, QWP, SQM, and QCT.

\begin{tabular}{lcccc}
\hline \hline & EQM & QWP & SQM & QCT \\
\hline$\sigma_{v^{\prime}=0, j^{\prime}=0}$ & 6.24 & 3.86 & 8.57 & 1.83 \\
$\sigma_{v^{\prime}=0, j^{\prime}=1}$ & 10.61 & 8.84 & 11.92 & 7.08 \\
$\sigma_{v^{\prime}=0, j^{\prime}=2}$ & 6.74 & 5.52 & 8.77 & 5.86 \\
$\sigma_{\sigma^{\prime}=0, j^{\prime}=3}$ & 0 & 0 & 0 & 0.74 \\
$\sigma_{v^{\prime}=0}$ & 23.60 & 18.22 & 29.25 & 15.51 \\
\hline \hline
\end{tabular}

not in phase with those exhibited by the EQM approach. The only significant discrepancy with the exact result is that the average value of the reaction probability for the lowest $J$ 's $(J<7)$ is about 0.5 in comparison with the corresponding average value of about 0.35 for the EQM calculation. Interestingly, the SQM opacity function is somewhat overestimating the exact reaction probabilities. The comparison between the reaction probabilities obtained by means of both methods (SQM and EQM) at the largest $J$ values indicates, nevertheless, that the EQM opacity function bears some statistical characteristics.

The ICSs calculated at this collision energy listed in Table II indicate that the preference for the $j^{\prime}=1$ state in the EQM calculations is also manifested in results obtained by means of the QWP, SQM, and QCT approaches. The SQM values are, however, slightly larger than the EQM ones and the QCT distribution, quite similar to the QWP result, differs from the exact ICSs in the underestimated population of $j^{\prime}$ $=1$ and the nonzero cross section found for $j^{\prime}=3$, which is not accessible quantum mechanically at this collision energy. The total ICSs indicate that the excess in reactivity in the SQM calculation with respect to the EQM value $(\sim 24 \%)$ is larger than at the higher collision energy $E_{c}=0.524 \mathrm{eV}$ $(\sim 10 \%)$. The QCT ICS, on the other hand, as in the other energy under study, is smaller $(\sim 34 \%)$ than the EQM value, but the comparison certainly improves the disagreement found at $E_{c}=0.524 \mathrm{eV}(\sim 44 \%)$. Analogously, the value obtained with the QWP approach, also below the exact ICS, represents a significant improvement $(\sim 23 \%)$ in the comparison with the EQM result with respect the observed values at $E_{c}=0.524 \mathrm{eV}$ (the QWP ICS is $\sim 37 \%$ smaller than the exact result at that energy).

Despite the above mentioned discrepancies between the exact and statistical values of the ICSs, the comparison between the corresponding DCSs, shown in Fig. 11, reveals interesting features. The peaks at the forward and backward scattering directions in the EQM DCSs at $0.1 \mathrm{eV}$ collision energy are more pronounced than those found at the higher collision energy considered in Sec. IV B 3. Moreover, the degree of asymmetry with respect to the sideways direction is also larger than in the previous case. Despite this fact, the SQM result reproduces perfectly the EQM value in the backward direction, although it does not give a good account for the dramatic preference for forward direction in the EQM case. The QCT and QWP approaches also fail to describe the extreme behavior observed in the EQM DCS.

The analysis of the calculated state-to-state DCSs, 


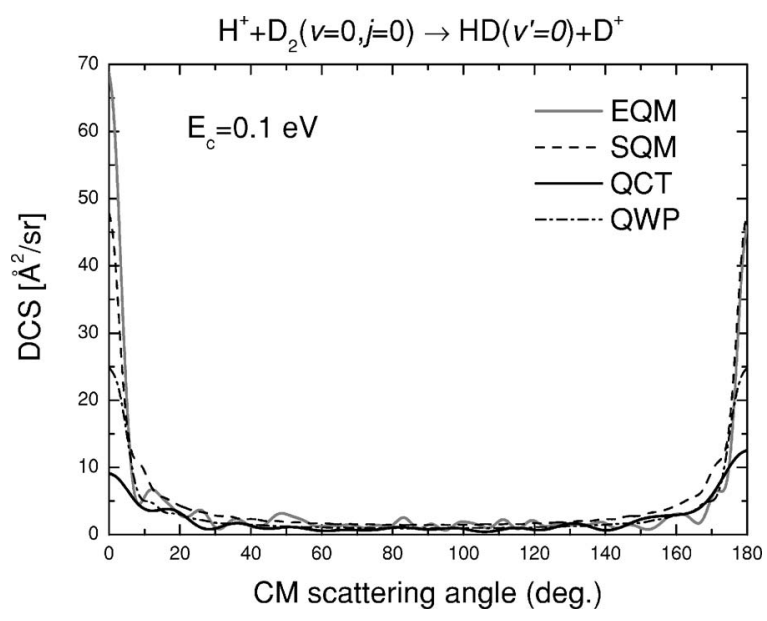

FIG. 11. Total differential cross section (in $\AA^{2} / \mathrm{sr}$ ) calculated at $0.1 \mathrm{eV}$ collision energy for the $\mathrm{H}^{+}+\mathrm{D}_{2}(v=0, j=0)$ reaction using the EQM (grey solid line), QWP (dashed-dotted line), SQM (dashed line), and QCT (solid line) methods.

shown in Fig. 12, reveals that most of the behavior found for the total DCS at $E_{c}=0.1 \mathrm{eV}$ is due to the $\mathrm{H}^{+}+\mathrm{D}_{2}(v=0, j$ $=0) \rightarrow \mathrm{HD}\left(v^{\prime}=0, j^{\prime}=0\right)+\mathrm{D}^{+}$reaction. Thus, the EQM DCS for $j^{\prime}=0$ exhibits most of the basic features of the total DCS and the discrepancies among the different theoretical approaches remain as well for this specific transition. From the inset of the top panel of Fig. 12, where the DCSs around the backward direction is magnified, the most important disagreements between the QCT and QWP DCS and those obtained with the EQM and SQM methods are clear. For the two other final states, $j^{\prime}=1$ and 2 , the agreement among the different theoretical approaches is better.

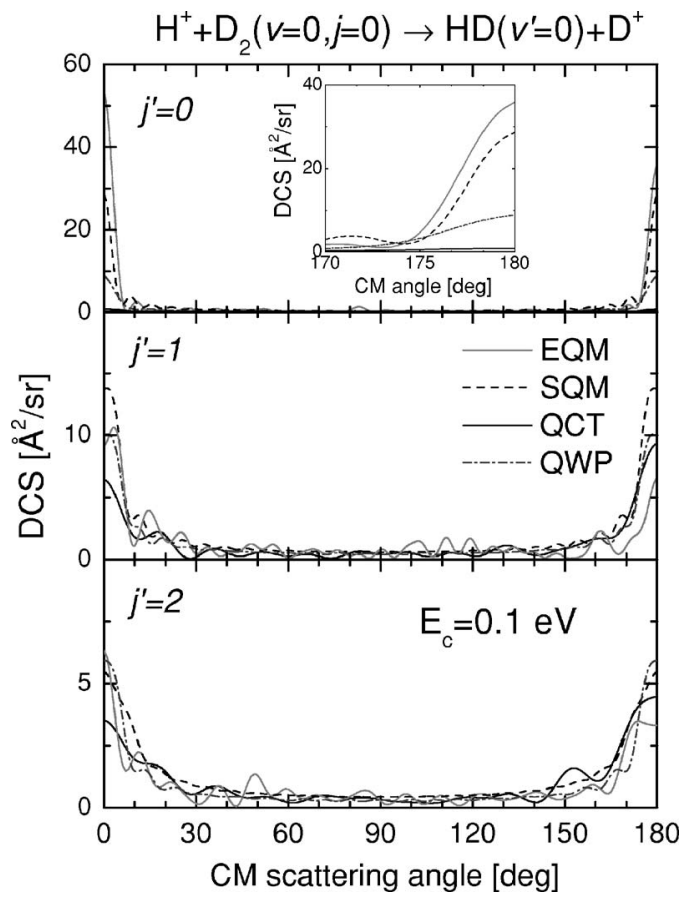

FIG. 12. Rotationally state resolved differential cross sections (in $\AA^{2} / \mathrm{sr}$ ) calculated at $0.1 \mathrm{eV}$ collision energy for the $\mathrm{H}^{+}+\mathrm{D}_{2}(v=0, j=0) \rightarrow \mathrm{HD}\left(v^{\prime}\right.$ $\left.=0, j^{\prime}=0-3\right)+\mathrm{D}^{+}$reactions by means of the EQM (grey solid line), QWP (dashed-dotted line), SQM (dashed line), and QCT (solid line) methods. The inset in the top panel shows a magnification of the backward region for the reaction yielding $\operatorname{HD}\left(v^{\prime}=0, j^{\prime}=0\right)$.

\section{DISCUSSION}

As already mentioned in Sec. I, the ion-diatom reactive collisions in the $\mathrm{H}_{3}^{+}$system, and its corresponding isotopic variants, are thought to occur via a complex-forming mechanism at low collision energies, whereas a direct reaction mechanism may play some role as collision energy increases. $^{6-9,11,26}$ It is hard to extract definitive conclusions in this sense from the present calculations at $E_{c}=0.1$ and $0.524 \mathrm{eV}$. The EQM results indicate that oscillations in the total reaction probability at both energies are similar. In addition, the degree of asymmetry observed in the corresponding DCSs is analogous $(\sim 1.55$ at $0.1 \mathrm{eV}$ and $\sim 1.2$ at $0.524 \mathrm{eV})$. Therefore, a not very different behavior is found at these two collision energies.

An indication of the existence of a transition in the reaction mechanism as a function of energy for the title reaction would be a distinct performance of the statistical approach to describe the main features observed in the exact results. In this sense, it is true that the SQM DCS seems to give a better description of the EQM DCS at the lower collision energy. Whereas at $0.1 \mathrm{eV}$ collision energy, both sideways and the peak at the backward scattering direction of the EQM DCS are clearly well reproduced by the SQM method, the forward and backward peaks of the DCS at $E_{c}$ $=0.524 \mathrm{eV}$ are manifestly overestimated. However, the SQM value for the total ICS at the higher energy differs less from the exact result than in the case of $E_{c}=0.1 \mathrm{eV}$.

The issue itself of whether or not the reaction can be well described by statistical means deserves some discussion. In general, the present study indicates that the SQM method manages to account for most of the main aspects reflected in the EQM results. For instance, the statistical prediction clearly constitutes the average of reaction probabilities examined at $J=0$. In view of the EQM vs SQM comparison at $J=40$ in the vicinity of $E_{c}=0.524 \mathrm{eV}$, it is likely that the agreement with the exact opacity function might improve at slightly different collision energies. Statistical and exact rotationally resolved ICSs are also in fairly good agreement. In the case of the QCT treatments, the failure of the traditional approach is remarkably well solved when the SQCT method is applied, leaving then no room to invoke only purely QM effects as a reason for the observed discrepancies between the QCT and EQM results.

At this stage we do not have a complete answer for the behavior of the reactivity as described by means of the QWP and QCT methods for large values of $J$. The remarkable thing is that both methods exhibit analogous failures despite the fact that rotational barriers are treated differently in each case: the QWP method, according with the ACS approximation simply considers a single helicity channel (that leading to the minimum barrier for given values if $J$ and $j$ ) in contrast with the classical treatment given within the QCT approach. In the case of the wave packet calculation, it has been found that the description of the formation of rotationally excited $\operatorname{HD}\left(v^{\prime}=0, j^{\prime}\right)$ product molecules with $j^{\prime}>2$ seems not to be dynamically correct. As mentioned in the previous section, it might be related to the ACS approximation employed in the present study, which favors the ener- 
getically lowest $\Omega^{\prime}$ to form products. For the QCT approach, it is not easy to understand the reason why trajectories which get trapped at some point in the potential well region suddenly leave the complex-forming region back to the reagents when the total angular momentum increases beyond certain values. One could think that somehow the energy released onto rotation of the intermediate complex hinders the system to fragmentate. The different performance of usual QCT approaches on the title reaction in comparison with statistical techniques, which has been also subject of previous studies, ${ }^{29,30,33}$ may have its origin in possible deficiencies of the classical (and also the ACS QWP) method to give account for the density of states at both sides of the rotational barriers which act as effective bottlenecks to favor the fragmentation.

The present study on the $\mathrm{H}^{+}+\mathrm{D}_{2}$ reaction seems to suggest some interesting isotopic effects in comparison with the $\mathrm{H}^{+}+\mathrm{H}_{2}$ analog, which was treated on a previous work. ${ }^{21}$ For instance, the extremely oscillating behavior with respect to the final rotational state found on the ICSs of the $\mathrm{H}^{+}+\mathrm{H}_{2}$ reaction at $E_{c}=0.44 \mathrm{eV}$ does not appear in the title reaction. Moreover, the DCSs exhibit, in the present case, a markedly different aspect, with not such an extreme preference for the forward and backward scattering directions. In this sense, such feature was more connected with a statistical dynamics for the $\mathrm{H}^{+}+\mathrm{H}_{2}$ reaction, as revealed by the good degree of agreement found between the EQM and SQM approaches. For the $\mathrm{H}^{+}+\mathrm{D}_{2}$ reaction, on the contrary, the preference for the $\theta \sim 0^{\circ}$ and $\theta \sim 180^{\circ}$ scattering directions is more apparent at $0.1 \mathrm{eV}$ collision energy. It would be interesting to investigate further these discrepancies between both reactions by using a different PES, since an interesting dependence on both the precise collision energy and the surface employed has been observed. Work in this direction is currently in progress.

An interesting issue is the comparison with the existing experimental results on the title reaction. The present study constitutes, to our knowledge, the first attempt to reproduce by means of QM approaches the substantial body of experimental results for the $\mathrm{H}^{+}+\mathrm{D}_{2}$ reactive system. The extensive theoretical analysis carried out in the present work reveals that, in general, the agreement with the existing measurements is not bad. Unfortunately, up to now the experiments have not provided too much information at the vicinity of the forward and backward scattering directions, which is where the results of the various theoretical methods differ at most. Due to this limitation on the angular range resolved by the experiments, it is difficult to conclude anything regarding the asymmetry or symmetry of the measured DCS. The EQM DCS, however, exhibits a slight preference for the backward direction at $E_{c}=0.524 \mathrm{eV}$. The favored peak at the other collision energy investigated, $0.1 \mathrm{eV}$, is on the contrary, the forward direction.

The simulations performed to obtain the KES indicate that the present calculations qualitatively describe the experimental findings. Although predictions on the intensities of the existing peaks in the measured KES from the different methods do not completely agree, dramatic discrepancies are not observed. Significant contributions from inelastic processes, also present in the measured spectra, jeopardize a complete quantitave agreement. Surely, new measurements could elucidate the adequacy of the present results to explain the dynamics of the process.

\section{CONCLUSIONS}

The dynamics of the $\mathrm{H}^{+}+\mathrm{D}_{2}(v=0, j=0) \rightarrow \mathrm{HD}+\mathrm{D}^{+}$reaction have been examined in detail by means of a series of theoretical approaches: a time independent exact quantum mechanical method, a quantum wave packet approach within the adiabatic centrifugal sudden approximation, a statistical quantum model, and a quasiclassical trajectory calculation. The investigation at 0.1 and $0.524 \mathrm{eV}$ collision energies has enabled the possibility of probing the transition from a complex-forming dynamics at a low energy regime and a direct reaction pathway at high energy. The slightly better agreement found between the statistical approach with the exact calculations could be interpreted as indications of such evolution in the overall reaction mechanism but it is hard to extract any conclusive evidences in this regard. The failure of the quasiclassical trajectory method at large values of the total angular momentum cannot be attributed to tunneling or similar purely quantum mechanical effects since previous studies performed with a statistical-based approach which also makes use of such trajectories are in good agreement with the exact results. In that sense, the apparent inadequacy of the approximate quantum wave packet calculation to correctly describe the dynamics at large $J$ 's is interpreted as due to the use of the adiabatical centrifugal sudden approximation.

The comparison between present theoretical findings and previous measurements reveal a fairly good description of the experiment, despite the forward and backward scattering directions in differential cross sections and spectra, the angular regions in which discrepancies among predictions by the different approaches are more significant, are not well described in the experimental data. The simulations of the kinetic energy spectra indicate that all methods yield a reasonable qualitative description of the existing experimental data.

\section{ACKNOWLEDGMENTS}

N.B. acknowledges a postdoctoral fellowship by the Spanish Ministry of Education and Science (MEC) under the program "Estancias de jóvenes doctores y tecnólogos extranjeros en España." This work has been funded by MEC of Spain under Grant Nos. FIS2004-02461, CTQ2004-02415, and CTQ2005-08493-C02-01 and from the Comunidad de Madrid under the Contrato Programa Comunidad de MadridUniversidad Complutense de Madrid (Grant No. 910729). A.T. and E.W. thank the EPSRC for financial support. The EQM calculations were performed on vector supercomputers, through a grant from the "Institut du Développment des Ressources en Informatique Scientifique" (IDRIS) in Orsay (France). E.C.N. T.G.L., and O.R. would also like to acknowledge the use of the computer resources, technical expertise, and assistance provided by the Red Española 
de Supercomputación (Barcelona Supercomputing Center and Centro de Supercomputación y Visualización de Madrid).

${ }^{1}$ D. Dai, C. C. Wang, S. A. Harich, H. Song, M. Hayes, R. T. Skodje, X. Wang, D. Gerlich, and X. Yang, Phys. Rev. Lett. 95, 013201 (2005).

${ }^{2}$ E. Wrede, L. Schnieder, K. Seekamp-Schnieder, B. Niederjohann, and K. H. Welge, Phys. Chem. Chem. Phys. 7, 1577 (2005).

${ }^{3}$ H. Song, D. Dai, G. Wu, C. C. Wang, S. A. Harich, M. Y. Hayes, X. Wang, D. Gerlich, X. Yang, and R. T. Skodje, J. Chem. Phys. 123 074314 (2005).

${ }^{4}$ M. Y. Hayes and R. T. Skodje, J. Chem. Phys. 126, 104306 (2007).

${ }^{5}$ J. C. Tully and R. K. Preston, J. Chem. Phys. 55, 562 (1971).

${ }^{6}$ J. R. Krenos, R. K. Preston, R. Wolfgang, and J. C. Tully, J. Chem. Phys. 60, 1634 (1974).

${ }^{7}$ D. Gerlich, U. Nowotny, Ch. Schlier, and E. Teloy, Chem. Phys. 47, 245 (1980).

${ }^{8}$ Ch. Schlier and U. Vix, Chem. Phys. 95, 401 (1985).

${ }^{9}$ C. G. Schlier and U. Vix, Chem. Phys. 113, 211 (1987).

${ }^{10}$ O. Brass and C. Schlier, J. Chem. Soc., Faraday Trans. 89, 1533 (1993)

${ }^{11}$ A. Ichihara, T. Shirai, and K. Yokoyama, J. Chem. Phys. 105, 1857 (1996).

${ }^{12}$ M. Chajia and R. D. Levine, Phys. Chem. Chem. Phys. 1, 1205 (1999).

${ }^{13}$ T. Takayanagi, Y. Kurosaki, and A. Ichihara, J. Chem. Phys. 112, 2615 (2000).

${ }^{14}$ A. Ichihara, O. Iwamoto, and R. K. Janev, J. Phys. B 33, 4747 (2000).

${ }^{15}$ V. G. Ushakov, H. Nobusada, and V. I. Osherov, Phys. Chem. Chem. Phys. 3, 63 (2001).

${ }^{16}$ H. Kamisaka, W. Bian, K. Nobusada, and H. Nakamura, J. Chem. Phys. 116, 654 (2002).

${ }^{17}$ N. Marković and G. D. Billing, Chem. Phys. Lett. 248, 420 (1996).

${ }^{18}$ T.-S. Chu and K.-L. Han, J. Phys. Chem. A 109, 2050 (2005).

${ }^{19}$ R.-F. Lu, T.-S. Chu, and K.-L. Han, J. Phys. Chem. A 109, 6683 (2005).

${ }^{20}$ T. González-Lezana, A. Aguado, M. Paniagua, and O. Roncero, J. Chem. Phys. 123, 194309 (2005).

${ }^{21}$ T. González-Lezana, O. Roncero, P. Honvault, J.-M. Launay, N. Bulut, F. J. Aoiz, and L. Bañares, J. Chem. Phys. 125, 94314 (2006).

${ }^{22}$ I. G. Csizmadia, J. C. Polanyi, A. C. Roach, and W. H. Wong, Can. J. Chem. 47, 4097 (1969).

${ }^{23}$ J. Krenos and R. Wolfgang, J. Chem. Phys. 52, 5961 (1970).

${ }^{24}$ R. Preston and J. C. Tully, J. Chem. Phys. 54, 4297 (1971).

${ }^{25}$ M. G. Holliday, J. T. Muckerman, and L. Friedman, J. Chem. Phys. 54, 1058 (1971)

${ }^{26}$ Ch. Schlier, U. Nowotny, and E. Teloy, Chem. Phys. 111, 401 (1987).

${ }^{27}$ E. J. Rackham, F. Huarte-Larrañaga, and D. D. Manolopoulos, Chem. Phys. Lett. 343, 356 (2001).

${ }^{28}$ E. J. Rackham, T. González-Lezana, and D. E. Manolopoulos, J. Chem. Phys. 119, 12895 (2003).

${ }^{29}$ F. J. Aoiz, T. González-Lezana, and V. Sáez Rábanos (unpublished).

${ }^{30}$ F. J. Aoiz, V. Sáez Rábanos, T. González-Lezana, and D. E. Manolopoulos, J. Chem. Phys. 126, 161101 (2007).
${ }^{31}$ L. Bonnet, P. Larrégaray, J.-C. Rayez, and T. González-Lezana, Phys. Chem. Chem. Phys. 8, 3951 (2006).

${ }^{32}$ L. Bonnet, P. Larrégaray, and J.-C. Rayez, Phys. Chem. Chem. Phys. 9, 3228 (2007).

${ }^{33}$ M. Berblinger and C. Schlier, J. Chem. Phys. 101, 4750 (1994).

${ }^{34}$ A. Aguado, O. Roncero, C. Tablero, C. Sanz, and M. Paniagua, J. Chem. Phys. 112, 1240 (2001).

${ }^{35}$ P. Honvault and J.-M. Launay, in Theory of Chemical Reaction Dynamics, edited by A. Lagana and G. Lendvay (Kluwer, Dordrecht, 2004).

${ }^{36}$ P. Honvault, J.-M. Launay, J. Chem. Phys. 111, 6665 (1999); N. Balucani, L. Cartechini, G. Capozza, E. Segoloni, P. Casavecchia, G. G. Volpi, F. J. Aoiz, L. Bañares, P. Honvault, and J.-M. Launay, Phys. Rev. Lett. 89, 013201 (2002)

${ }^{37}$ P. Soldan, M. T. Cvitas, J. M. Hutson, P. Honvault, and J.-M. Launay, Phys. Rev. Lett. 89, 153201 (2002); M. T. Cvitas, P. Soldan, J. M. Hutson, P. Honvault, and J.-M. Launay, ibid. 94, 033201 (2005).

${ }^{38}$ D. E. Manolopoulos, J. Chem. Phys. 85, 6425 (1986).

${ }^{39}$ Y. Huang, D. J. Kouri, and D. K. Hoffman, J. Chem. Phys. 101, 10493 (1994).

${ }^{40}$ V. A. Mandelshtam and H. S. Taylor, J. Chem. Phys. 103, 2903 (1995).

${ }^{41}$ Y. Huang, S. S. Iyengar, D. J. Kouri, and D. K. Hoffman, J. Chem. Phys. 105, 927 (1996).

${ }^{42}$ G.-J. Kroes and D. Neuhauser, J. Chem. Phys. 105, 8690 (1996).

${ }^{43}$ R. Chen and H. Guo, J. Chem. Phys. 105, 3569 (1996).

${ }^{44}$ S. K. Gray and G. G. Balint-Kurti, J. Chem. Phys. 108, 950 (1998).

${ }^{45}$ S. Gómez-Carrasco and O. Roncero, J. Chem. Phys. 125, 054102 (2006).

${ }^{46}$ G. G. Balint-Kurti, R. N. Dixon, and C. C. Marston, J. Chem. Soc., Faraday Trans. 86, 1741 (1990).

${ }^{47}$ R. N. Zare, Angular Momentum (Wiley, New York, 1988).

${ }^{48}$ T. González-Lezana, Int. Rev. Phys. Chem. 26, 29 (2007), and references therein.

${ }^{49}$ F. J. Aoiz, L. Bañares, and V. J. Herrero, J. Chem. Soc., Faraday Trans. 94, 2483 (1998).

${ }^{50}$ L. Bañares, F. J. Aoiz, P. Honvault, B. Bussery-Honvault, and J.-M. Launay, J. Chem. Phys. 118, 565 (2003).

${ }^{51}$ L. Bañares, F. J. Aoiz, P. Honvault, and J.-M. Launay, J. Phys. Chem. A 108, 1616 (2004).

${ }^{52}$ L. Bonnet and J. -C. Rayez, Chem. Phys. Lett. 277, 183 (1997); ibid. 397, 106 (2004).

${ }^{53}$ L. Schnieder, K. Seekamp-Rahn, E. Wrede, and K. H. Welge, J. Chem. Phys. 107, 6175 (1997).

${ }^{54}$ We have observed that the units of the QCT DCSs calculated at $E_{c}$ $=0.524 \mathrm{eV}$ shown in Fig. 4 of Ref. 3 are likely to be $a_{0}^{2} / \mathrm{sr}$ instead $\AA^{2} / \mathrm{sr}$ as indicated in the corresponding caption to the figure. If so, those results and the present QCT DCSs are in good agreement within the angular range considered in Ref. 3, although both calculations were carried out in different potential energy surfaces. Moreover, the corresponding ICS shown in Fig. 2 of the work by Song et al. at the same collision energy would be then consistent with the DCS shown in Fig. 3 of the same work.

${ }^{55}$ Note that the laboratory angles reported here include corrections from an angle calibration measurement whereas those stated in Ref. 2 are nominal angles. 